Sharif University of Technology
Scientia Iranica
Transactions E: Industrial Engineering
http://scientiairanica.sharif.edu
IRAN I CA

\title{
A novel fuzzy bi-objective vehicle routing and scheduling problem with time window constraint for a distribution system: A case study
}

\author{
A. Esmaeilidouki ${ }^{\mathrm{a}, *}$, M. Mahzouni-Sani ${ }^{\mathrm{b}}$, A. Nikhalat Jahromi ${ }^{\mathrm{c}}$, and F. Jolai ${ }^{\mathrm{d}}$ \\ a. Department of Industrial Engineering, Firoozkooh Branch, Islamic Azad University, Firoozkooh, Iran. \\ b. Department of Electrical Engineering, Urmia University, Urmia, Iran. \\ c. Department of Industrial Engineering, University of Science and Culture, Tehran, Iran. \\ d. School of Industrial Engineering, College of Engineering, University of Tehran, Tehran, Iran.
}

Received 13 December 2018; received in revised form 13 October 2019; accepted 18 February 2020

\author{
KEYWORDS \\ Hazardous material \\ distribution problem; \\ Vehicle routing and \\ scheduling; \\ Fuzzy inference \\ system and fuzzy \\ failure mode and \\ effects analysis; \\ Time window \\ constraint; \\ Fuzzy goal \\ programming.
}

\begin{abstract}
During the transportation of dangerous, the risk is an important factor that should be considered due to the potentially serious consequences of the accident. Regardless of risks, time is the primary issue that should be considered in the transportation of hazardous materials. This paper introduces a bi-objective model of vehicle routing and scheduling for hazardous material allocation problems under fuzzy conditions to minimize the total allocation time and risk. In the proposed model, the fuzzy inference system and fuzzy failure mode and effects analysis are used for the first time to identify and calculate high-level risks, instead of the previous simple methods. Moreover, the Jimenez method and fuzzy goal programming are respectively utilized to convert the fuzzy biobjective model into the same crisp and single-objective one. In addition, in order to deal with the NP-hardness of large-scale problems, two meta-heuristic algorithms, invasive weed optimization, and genetic algorithm, are used, and multiple sensitivity analyses are performed to prove the effectiveness of the proposed method. The performance of the proposed algorithms is also assessed through a comparative study. Finally, the proposed model is applied to a real case study to prove the validity of the model.
\end{abstract}

(C) 2021 Sharif University of Technology. All rights reserved.

\section{Introduction}

Nowadays, the dynamics of global industrialization have brought a higher rate of industrials activities and hazardous materials (HAZMAT) transportations [1].

*. Corresponding author. Tel.: +1 2508787975

E-mail addresses: esmaili.ayda1@gmail.com ( $A$.

Esmaeilidouki); mahdimahzouni@gmail.com (M.

Mahzouni-Sani); abbasnikhalat@yahoo.com (A. Nikhalat

Jahromi);fjolai@ut.ac.ir (F.Jolai)

doi: $10.24200 /$ sci.2020.52461.2727
According to the US Census Bureau's 2012 Commodity Flow report, 2.5 billion tonnes of HAZMAT were transported in the United States in 2012, a significant rise of $15.6 \%$ in HAZMAT transportation [2]. Also, most materials transported by truck, ship, train, and plumbers are flammable, explosive, toxic, and radioactive, and these materials are potentially dangerous. As declared by the US Department of Transportation, the risk of having HAZMAT transportation incidents has increased so that in 2009 there were 14,816 HAZMAT incidents resulting from different transportation modes whereas 17,459 accidents were recorded in 2012, re- 
sulting in 7 fatalities, 152 injuries, and $\$ 68,045,434$ damages [3]. Therefore, due to the dangerous nature of hazardous material, the transportation risk of HAZMAT and the associated consequences of their events attract both governments, and the public attention, and the risk factor is the crucial factor that should be considered in HAZMAT transportation.

Moreover, today to find the best practice regarding the vehicle routing and scheduling which could ensure the customers satisfaction to the best come to be the major concern of researchers. Today, to find the best routes and schedules for servicing the customers come to be the major concern of researchers [4-6]. Indeed, routing decisions identify the best routes for transferring Hazmat via the transportation system [7], and scheduling decisions provide the optimal scheduling for multiple vehicles to deliver a certain product to a particular set of consumers. Besides, although many attempts are made to reduce the harmful effects of HAZMAT incidents, the desired objectives are not met and serious adverse consequences are observed. Therefore, the appropriate HAZMAT routing and the secure network design are needed to distribute these materials so that they could reduce the potential adverse effects of transportation. Although finding the best vehicle route can significantly improve customer service, many researchers did not consider these key decisions in their research. In other words, due to the multiple objectives of dangerous materials distribution, the provision of a real-world model can improve the level of transportation safety.

The dynamic nature of some parameters like time is an inevitable part of vehicle routing and scheduling networks since it can be changed due to uncertain circumstances and can affect the vehicle routing and scheduling network design. Therefore, uncertainties are considered in the literature of vehicle routing and scheduling, and they are investigated in different ways. The topic investigated in this regard include stochastic [8,9], fuzzy [9-12], robust [13], and robust/fuzzy [14] parameters.

In the proposed model, one of the goals is to minimize the total scheduled distribution time of Hazardous Material Distribution Problems (HMDP). Therefore, careful consideration of the proper scheduling of HAZMAT in the dangerous materials transportation system has raised an important question. In addition, the problem under consideration is defined as routing vehicles to determine the best transportation route to transport products from the warehouse to the customer nodes. On the other hand, risk is one of the most important criteria for selecting the routes of HMDP which makes this problem more serious than others. Then, the other aim of the proposed model is to minimize the maximum total Risk Priority Number $(\mathrm{RPN})$ in the hazardous transportation system. In fact, although the models for calculating risk values in the literature are different, a new risk calculation method is proposed as one of the most basic methods to determine the total risk. This means that firstly, the high-level risks are identified and calculated by using the inference system and fuzzy Failure Mode and Effects Analysis (FMEA), and then the calculated risks are minimized by the objective function of the presented model. This method of determining risks is new and proposed for the first time in this study. Besides risk, minimizing distribution time is the main objective that should be considered in any dangerous materials transportation. Numbers of attributes are available in the literature, most of which focus on finding the shortest paths for a given origin-destination pair. However, in reality, the HAZMAT transportation problem particularly based on a land mode like a typical vehicle routing problem calls for the determination of a set of optimal routes to be traveled by a fleet of vehicles, thus satisfying the demand and time window constraints of a pre-specified number of customers.

The purpose of this paper is to develop a novel mixed-integer non-linear programming model to design a vehicle routing and scheduling network for HAZMAT under fuzzy conditions. Indeed, the proposed model tries to minimize the total distribution time and the maximum total RPN between two nodes of the HAZMAT transportation system. Furthermore, the proposed model integrates routing decision-making with scheduling decision-making. The main contributions of the present study, which distinguish it from the previous studies in this area, are mentioned as below:

- Developing a novel bi-objective, vehicle routing and scheduling model of HAZMAT transportation system by considering the dynamic nature of time;

- Proposing a time function that attempts to minimize the total distribution time of the vehicle in the HAZMAT transportation process;

- Utilizing a new two-phase framework which includes fuzzy FMEA and Fuzzy Inference System (FIS) to identify the high-level risks in calculating the total risks imposed to the hazardous products transportation system;

- Applying two meta-heuristic methods called Invasive Weeds Optimization (IWO) and Genetic Algorithm (GA) for successfully solving the large-sized problems in an acceptable time, and implementing the presented model in a real case study.

The remainder of this study is expressed as follows: Section 2 reviews the relevant literature. In Section 3, the problem is described, and the mathematical model we developed is explained. Section 4 is devoted to the solution method. The results of the computational experiments and sensitivity analyses, along with the 
case study, are described in Section 5. Finally, conclusions and outlines of future studies are given in the last section.

\section{Literature review}

Several studies have considered the Vehicle Routing Problem with Time Window (VRPTW) model. Indeed, The VRPTW is a variant of VRP, which attempts to determine a set of vehicle routes that involve a single depot, a fleet of identical vehicles stationed at the depot, and a set of customers who require delivery of goods from the depot [15]. Although some researchers such as Taniguchi et al. [16] have tried to propose single-objective mathematical models of the problem, other researchers have also introduced some multi-objective models. For example, Zhang et al. [17] presented a new VRPT model for HAZMAT transportation by adding upper load bounds on the road segments and solved it by developing a sophisticated Tabu Search (TS) algorithm with a dynamic penalty mechanism to obtain good solutions. Besides, Pradhananga et al. [5] try to overcome the problem by making the route selection and routing phase at the same phase so as to provide opportunities for all non-dominant routes in the route selection process. They solved the presented multi-objective model by proposing the new method, which works based on an ant colony algorithm to make both route selection and routing phases in the same phase to propose the optimal Pareto solutions.

Various studies have recommended proper approaches to solve the VRPT problem [11,12,18-20]. Also, as VRPT is an NP-hard problem with complicated calculation, many studies have focused on heuristic algorithms [9,21-23]. As one, Zografos and Androutsopoulos [24] investigated the VRPTW of HAZMAT transportation by proposing a bi-objective model and applied a weighting method to convert the model into a single-objective model. Then a heuristic algorithm was applied to select the best routes of network nodes, and finally, the customer service sequences were determined by Dijkstra shortest path algorithm. Also, the bi-objective model of HMDP is proposed by Androutsopoulos and Zografos [4], and it is converted to the single-objective model through the weighted sum method. They consider the intermediate stop in their heuristic approach and in order to prove that the computation time is not the inhibitor factor for using this algorithm, the proposed algorithm is implemented on several real samples.

In most cases, heuristic algorithms are only able to solve small-sized problems whereas most practical applications usually involve a large number of customers. Hence, some researchers tried to use metaheuristic algorithms in their studies [20,25-29]. The three-objective mathematical model proposed by Jalili Ball et al. [30] minimizes the total costs (cost of transportation and cost of delay) and total risks due to the time window limitation and the environmental factors so as to restore the balance of each vehicle assigned route. Then, the presented model is solved by two meta-heuristic algorithms, Non-dominated Sorting Genetic Algorithm II (NSGA-II) and Multi-Objective Vibration Damping Optimization (MOVDO), respectively. Finally, by solving 15 examples of different dimensions, the efficiency and effectiveness of these algorithms in solving the major example problems in a short period of time are confirmed. Besides, Alvarenga et al. [31] proposed a two-stage heuristic approach for solving the VRPTW. Their approach was involving an efficient GA and a set partitioning formulation. Their computational results outperformed the existing heuristic methods in terms of minimal travel distance. Another example is a new metaheuristic algorithm provided by Talarico et al., called ALNS (Ant colony heuristic algorithm with Large Neighborhood Search) [25]. This algorithm is proposed to solve the risk constrained cash-in-transit vehicle routing problem. Then, they have tested the proposed algorithm on small, medium, and large benchmark examples and compared it with all existing solution approaches in the literature to show the efficiency of the proposed method.

Risk is a progressively worldwide concern when it comes to the transportation of HAZMAT, attracting many researchers attention. The first challenge in determining risk is how to collect reliable information and categorize them according to their impact. Moreover, although there are many analytical approaches to hazardous materials transport risk, there is no agreement among researchers on how to model the associated risks [32]. Therefore, Erkut and Verter [32] studied a different risk model for Hazmat transportation by investigating the U.S. road network. They concluded that a solution might work in one model but not in another model. Also, Marhavilas et al. [33] developed a technique to analyze the risk by capturing real accident information. They used a mixture of qualitativequantitative and semi-quantitative methods to determine the quantitative risk of hazardous materials. They concluded that the quantitative methods have the highest relative frequency in comparison with the qualitative methods. Bonvicini et al. [34] utilized fuzzy logic to evaluate the uncertainty of the risk of transporting hazardous materials through roads and pipelines. Bubbico et al. [35] investigated information of road and rail accidents caused by the transportation of liquid gas and used the collected data to assess the risks of the case study. Topuz et al. [36] proposed a comprehensive quantitative multi-criteria method using Analytic Hierarchy Process (AHP) and fuzzy 
logic to assess the environmental and health risks associated with dangerous hazmat relating to industrial activities.

The complexity of the HAZMAT transportation vehicle routing and scheduling network increases the uncertainty of the design network. Therefore, in some studies $[8,10,11,13,14,27,37]$, solving uncertainty is considered an important problem. Androutsopoulos and Zografos [6] considered some route features such as risk, cost, and the total distribution time, also they emphasized the dependency on time and uncertainty. Also, Qureshi et al. [38] believed that travel time varies due to traffic congestion issues, accidents, and other similar factors. Meanwhile, if the routing is fixed due to the perspective of public transportation, unpredictable travel time changes will cause more cost increases and delays in the transportation and unloading of vehicles; it also depends on the exact travel time values (e.g. average travel time). Hence, they proposed a dynamic routing of the vehicle, considering a soft time window to solve this kind of problem.

The discussions mentioned above and Table 1 show that in the vehicle routing and scheduling literature, the topic under discussion is neglected.

The review of the literature reveals that the HAZMAT transportation network is not considered as a vehicle routing and scheduling problem. Hence in the present study, vehicle routing and scheduling of hazardous products are considered to make the model more realistic. advantages of Integrating routing and scheduling decisions have some advantages, however, only a few researchers have focused on proposing routing and scheduling models to distribute HAZMAT. In this paper, we propose a mathematical model of HAZMAT distribution through which routing and scheduling decisions can be made at the same time. Moreover, only a few researchers have focused on proposing a model of HMDP with time window constraints. Therefore, mathematical modeling in which the dynamic nature of time is considered can help to achieve a better design of the HAZMAT distribution network. In addition, the HAZMAT vehicle routing and scheduling network, although very important in such problems, lacks the concept of risk. In this research, we propose a new risk calculation framework to consider the risks imposed on the routing and scheduling of vehicles transported by HAZMAT. In addition, the relevant literature does not fully refer to the uncertainty in the HMDP. Therefore, the present research presents another gap in the available literature by taking into consideration the fuzzy parameters to refer to the uncertainty in a more practical way. This research applies the concept of fuzzy theory to consider the uncertainty of these parameters in the proposed mathematical model. In other words, as the required data for analyzing the useful parameters of the problem are limited and there is no reliable resource [34], we consider some parameters of the proposed model as fuzzy triangular numbers.

To overcome the shortcomings mentioned above and remove the gaps shown in Table 1, we proposed a new fuzzy mathematical model for an HMDP with time window constraint under fuzzy conditions to achieve the appropriate routing and scheduling of HAZMAT distribution. The proposed model can analyze the trade-off between the total distribution time and total risks imposed on the transportation network. Besides, two efficient algorithms namely IWO and GA, are utilized to solve the large-sized instances problem in an efficient way and in a reasonable time to obtain the near-optimal solutions.

\section{Problem explanation and mathematical modeling}

\subsection{Model framework}

In this section, a new HAZMAT mixed-integer mathematical model is proposed, which is completely different from the currently proposed hazardous materials model. Here, the main problem relating to vehicle routing and scheduling is to carry the HAZMAT from the depot to customer nodes through the available routes to meet the nodes requirements. The proposed model can be used to determine the safest routes. Through these routes, the time of the HAZMAT transportation from depot to customer nodes could be significantly reduced.

The proposed model presents a bi-objective vehicle routing and scheduling model in which two objectives are considered:

(i) Minimizing the total distribution time including total travel time between nodes and waiting time of the vehicle in customer nodes;

(ii) Minimizing the maximum of total RPN between origin-destination nodes in the hazardous transportation system.

In Subsection 3.2, the framework of estimating high-level risks by the FIS and fuzzy FMEA is explained. Although previous studies focused on simple risk calculation methods [39-41], the proposed framework introduced for the first time a new method to identify and rank the high-level risks imposed on hazardous product transportation systems. Also, the reason for using fuzzy FMEA in the proposed procedure is that the efficiency of this tool in risk calculation is proved by many previous studies [42-45].

The following assumptions are considered to formulate the model:

- Every vehicle begins its travel from the only available depot and finishes it at the same place; 
Table 1. Review of related studies.

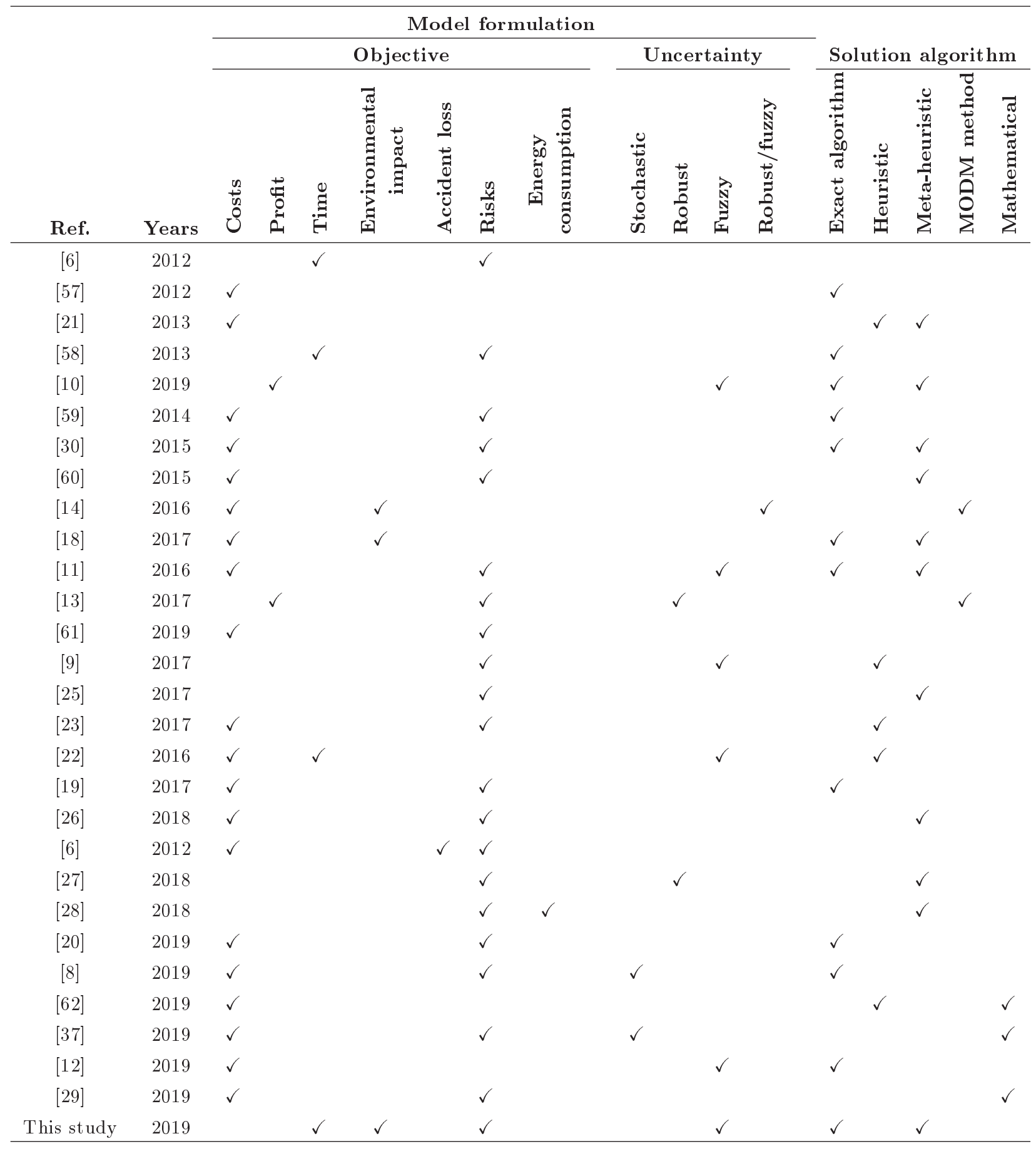

- Intermediate stops are only allowed in the customer nodes to deliver the goods on each route;

- Due to the nature of the HAZMAT in the distribution process, route characteristics such as risk and travel time have some degree of uncertainty;

- Every demand node should be served within a predetermined time window;
- The demand for each vehicle should not exceed the vehicle capacity;

- There is a time limit for using vehicles in the route;

- The time window limit determines the earliest and latest delivery time for each demand node;

- The vehicles are considered to have the same capacity. 


\subsection{FIS and fuzzy FMEA}

During the transportation of dangerous products, due to the negative consequences of transporting such materials, the risk is a substantial and important factor and should be considered. Meanwhile, these undesirable consequences caused interruptions and delay in the transportation process resulting in failure to deliver products in time, customer dissatisfaction, and loss of credibility. Based on the above, it seems necessary to apply appropriate tools to identify, classify and rank the high-level risks of hazardous product transportation systems. Therefore, various methods are proposed to calculate the risk value imposed on the hazardous products' transportation system [36,46,47].

In the present study, we proposed a novel framework to evaluate the hazardous product risk values as shown in Figure 1. In the first phase, the critical and effective risks are identified and classified, and then each risk is evaluated according to its severity $(S)$, the probability of occurrence $(O)$, and the detection likelihood $(D)$. In the problem under consideration, the required data for determining risks value are not available, or sometimes it is challenging to collect them, or in most cases, they can be found in the form of linguistic variables. Therefore, we utilized fuzzy FMEA to cope with these problems [48]. In this regard, the FIS can be considered, too.

The second phase is associated with the logic fuzzy implementation and includes the following steps:

1. Defining the membership functions and linguistic variables $S, O$, and $D$;

2. Construct the fuzzy rule base;

3. Construct the FIS;

4. Convert the Linguistic values of obtained RPN into the non-fuzzy values (defuzzification).

The output data of the previous phase is called RPN. In this study, the obtained RPN represents the high-level risk of each arc between the two nodes of the dangerous products transportation system. Finally, the proposed mixed integer programming can be used to minimize RPN to reduce the possibility of dangerous events in the final stage.

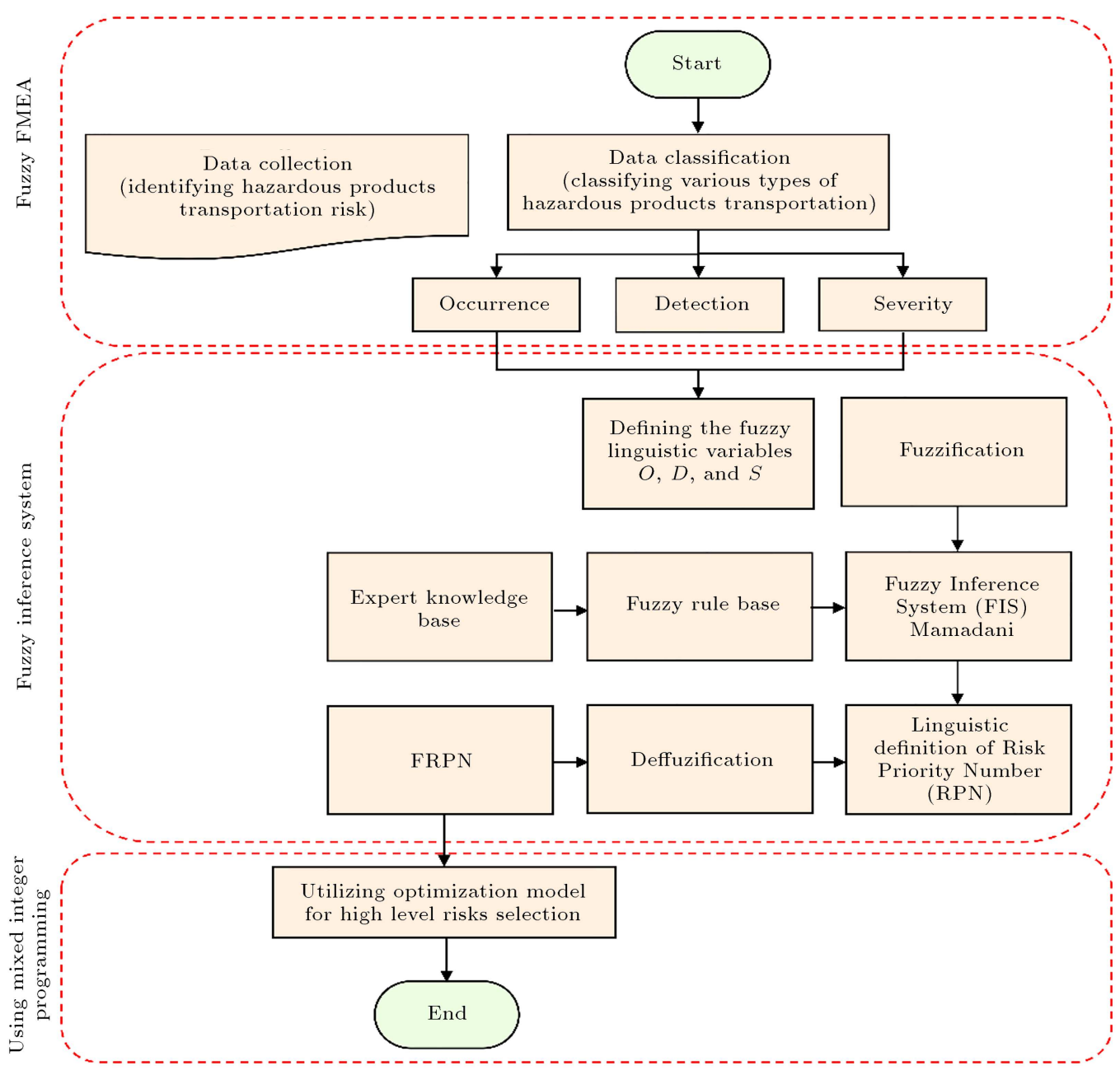

Figure 1. New framework of calculating the HAZMAT transportation risk. 
The indices, parameters and decision variables used in the mathematical model are as follow:

\section{Sets}

$i, j \quad$ Index of nodes

$k \quad$ Index of transportation vehicles

$l \quad$ Index of available routes

\section{Parameters}

$N V \quad$ Number of available vehicles

$M V \quad$ Maximum waiting time in customer node

MR Maximum allowable risk imposed on the hazardous material transportation system

$d_{i} \quad$ Customer demand amount in a node $i$ (weight/ number of product)

$C_{k} \quad$ Transportation capacity of vehicle $k$

$a_{i} \quad$ Start time of time window for node $i$

$b_{i} \quad$ End time of time window for node $i$

$T_{i}^{k} \quad$ Entry moment of the vehicle $k$ in node

$S_{i} \quad$ Service time of node $i$

$T_{D}^{k} \quad$ Departure moment of the vehicle $k$ from depot

$\tilde{t}_{i j}^{1} \quad$ Travel time between two nodes $i$ and $j$ through available route $l$

$R_{i j}^{l k} \quad$ Risk Priority Number (RPN) of the available route $l$ between nodes $i$ and $j$

\section{Decision variables}

$t_{i}^{w} \quad$ Waiting time for customer of node $i$

$Z_{1} \quad$ Total scheduled distribution time

$Z_{2} \quad$ Total maximum risk imposed on the transportation system

$X_{i j}^{l k} \quad 1$ if vehicle $k$ travel between two nodes $i$ and $j$ through the available route $l ; 0$ otherwise

The non-linear mixed integer multi-objective model for scheduling the HDMP is formulated as follows:

$$
\begin{aligned}
& \min Z_{1}=\sum_{i=0}^{n} \sum_{j=1}^{n+1} \sum_{l=1}^{\widehat{N}_{i j}} \sum_{k=1}^{N V} X_{i j}^{l k} \widetilde{t}_{i j}^{l}+\sum_{i=1}^{n} t_{i}^{w}, \\
& \max \quad Z_{2}=\sum_{i=0}^{n} \sum_{j=1}^{n+1} \sum_{l=1}^{\widehat{N}_{i j}} \sum_{k=1}^{N V} X_{i j}^{l k} R_{i j}^{l k},
\end{aligned}
$$

s.t.:

$$
\sum_{i=0}^{n} \sum_{l=1}^{\widehat{N}_{i j}} \sum_{k=1}^{N V} X_{i j}^{l k}=1 \quad j=1, \cdots, n
$$

$$
\begin{aligned}
& \sum_{i=0}^{n} \sum_{l=0}^{\widehat{N}_{i j}} X_{i p}^{l k}-\sum_{j=1}^{n+1} \sum_{l=1}^{\widehat{N}_{i j}} X_{p j}^{l k}=0 \\
& k=1, \cdots, N V, \quad p=1, \cdots, n, \\
& \sum_{j=1}^{n} \sum_{l=1}^{\widehat{N}_{o j}} X_{o j}^{l k}=1 \quad k=1, \cdots, N V \\
& \sum_{i=0}^{n} d_{i}\left(\sum_{j=1}^{n+1} \sum_{l=1}^{\widehat{N}_{i j}} X_{i j}^{l k}\right) £ C_{k} \quad k=1, \cdots, N V, \\
& T_{i}^{k}+s_{i}+X_{i j}^{l k}+\widetilde{t}_{i j}+t_{j}^{w}-T_{j}^{k} \leq\left(1-X_{i j}^{l k}\right) M \\
& i=1, \cdots, n ; \quad j=1, \cdots, n ; \quad k=1, \cdots, N V \\
& l=1, \cdots, \widehat{N}_{i j} \\
& T_{i}^{k}+s_{i}+X_{i j}^{l k}+\tilde{t}_{i j}+t_{j}^{w}-T_{j}^{k} \geq\left(1-X_{i j}^{l k}\right) M \\
& i=1, \cdots, n ; \quad j=1, \cdots, n ; \quad k=1, \cdots, N V \\
& l=1, \cdots, \widehat{N}_{i j} \\
& T_{D}^{k}+X_{o j}^{l k} \tilde{t}_{i j}+t_{j}^{w}-T_{j}^{k} \leq\left(1-X_{o j}^{l k}\right) M \\
& i=1, \cdots, n ; \quad j=1, \cdots, n ; \quad k=1, \cdots, N V \\
& l=1, \cdots, \widehat{N}_{i j} \\
& T_{D}^{k}+X_{o j}^{l k} \tilde{t}_{i j}^{l}+t_{j}^{w}-T_{j}^{k} \geq\left(X_{o j}^{l k}-1\right) M \\
& i=1, \cdots, n ; \quad j=1, \cdots, n ; \quad k=1, \cdots, N V \text {; } \\
& l=1, \cdots, \widehat{N}_{i j} \\
& a_{j} \leq \sum_{k=1}^{N V} T_{j}^{k} \leq b_{j} \quad i=1, \cdots, n ; \quad k=1, \cdots, N V \\
& T_{j}^{k} \leq\left(\sum_{j=1}^{n+1} \sum_{l=1}^{\widehat{N}_{i j}} X_{i j}^{l k}\right) M \quad k=1, \cdots, N V \\
& a_{0} \leq T_{k}^{D} \leq b_{0} \quad k=1, \cdots, N V, \\
& \sum_{j=1}^{n} t_{j}^{w} \leq M W \\
& \sum_{i=0}^{n} \sum_{j=1}^{n+1} \sum_{l=1}^{\widehat{N}_{i j}} \sum_{k=1}^{N V} X_{i j}^{l K} R_{i j}^{l k} \leq M R \\
& X_{i j}^{l k}=0, \quad i, j=0, n+1 ; \quad k=1, \cdots N V ; \\
& l=1, \cdots, \widehat{N}_{i j},
\end{aligned}
$$




$$
\begin{aligned}
& X_{i j}^{l k}=0, \quad i=j ; \quad k=1, \cdots, N V ; \quad l=1, \cdots, \widehat{N}_{i j} \\
& t_{i}^{w}, T_{i}^{k}, T_{i}^{D} \in R^{+} \quad i=1, \cdots, n ; \quad k=1, \cdots, N V, \\
& X_{i j}^{l k} \in\{0,1\} .
\end{aligned}
$$

The objective function (1) minimizes the total distribution time including total travel time between nodes and waiting time of the vehicle in customer nodes. Meanwhile, the service time is not considered in the waiting time when determining the customer nodes. The objective function (2) tends to minimize the maximum of total RPN in the hazardous transportation system. In fact, first, the high-level risks are identified and calculated by using the inference system and fuzzy FMEA, and then the calculated risks are minimized by objective function (2). Constraint (3) ensure that each node can only be transported and served by one vehicle once. Constraint (4) guarantees that if a vehicle enters the node, it must leave it. Constraint (5) specifies that each vehicle should start its travel from a depot, and Constraints (4) and (5) force the vehicle to exit the itinerary of the same depots. Constraint (6) implies that the customer demands served by each vehicle should not exceed the vehicle capacity. Constraints (7) to (10) guarantee that if the vehicle $k$ enters to node $j$ the service's start time of which equals to the summation of entry time in node $i$, travel time between nodes $i$ to $j$ through route $l$, and waiting time in node $j$. Under these constraints, the parameter $M$ is considered to be a large number, and its value depends on the travel time between the two nodes $i$ and $j$ and the time to enter each node $j$ (waiting time at the client node). Constraint (11) ensures that the entry time in each node should be within its specific time window. Constraint (12) indicates that the variable $T_{j}^{k}$ is a nonzero value if the vehicle $k$ serves the customer node $j$. Constraint (13) states that the departure time must be within $a_{0}$ and $b_{0}$. Constraints (14) indicate that the total waiting times in customer nodes should not exceed a predefined value. Constraint (15) Ensure that each distribution warehouse should only be assigned to one supplier. Constraints (16) to (19) are assigned to the number and types of parameters and decision variables.

\section{Solution method}

In this section, we proposed a two-phased approach to solve the proposed bi-objective model under a fuzzy condition. The first phase includes converting the fuzzy model into its equivalent crisp one using the Jimenez method [49] and solving the crisp model through the Fuzzy Goal Programming (FGP) method. In the literature, it is proved that vehicle routing and scheduling problems belong to the NP-hard classes [50].
On the other hand, solving large-sized problems in a reasonable time seems very difficult. Then, in the second phase, an effective meta-heuristic algorithm (i.e., the IWO) is applied to achieve the near-optimal solution of the proposed model. Finally, in Section 5 the numerical results obtained by applying IWO are compared with other meta-heuristic algorithms, such as GA. In addition, the steps of the presented hybrid method are shown in Figure 2.

\subsection{Equivalent auxiliary crisp model}

In this study, we use the Jimenez method to convert the constraints and objective functions into crisp functions. In other words, Eqs. (20) and (21) are utilized to convert the triangular fuzzy number $\widetilde{A}$ of constraints and objective functions as follows:

$$
\begin{aligned}
& E I(\widetilde{A})=\left[\alpha\left(\frac{A^{p}+A^{m}}{2}\right)+(1-\alpha) \frac{A^{m}+A^{o}}{2}\right], \\
& E V(\tilde{A})=\frac{A^{p}+2 A^{m}+A^{o}}{4},
\end{aligned}
$$

where $A^{o}, A^{m}, A^{p}$, and $\alpha$ are the lowest possible value, the most likely value, the maximum possible value, and the satisfaction degree, respectively.

As mentioned above, using Eq. (21), the objective functions (1) and (2) are equal to the following auxiliary crisp functions:

$$
\begin{aligned}
\min Z_{1}= & \sum_{i=0}^{n} \sum_{j=1}^{n+1} \sum_{l=1}^{\widehat{N}_{i j}} \sum_{k=1}^{N V} X_{i j}^{l k}\left(\frac{t_{i j}^{l p}+2 t_{i j}^{l m}+t_{i j}^{l o}}{4}\right) \\
& +\sum_{i=1}^{n} t_{i}^{w},
\end{aligned}
$$

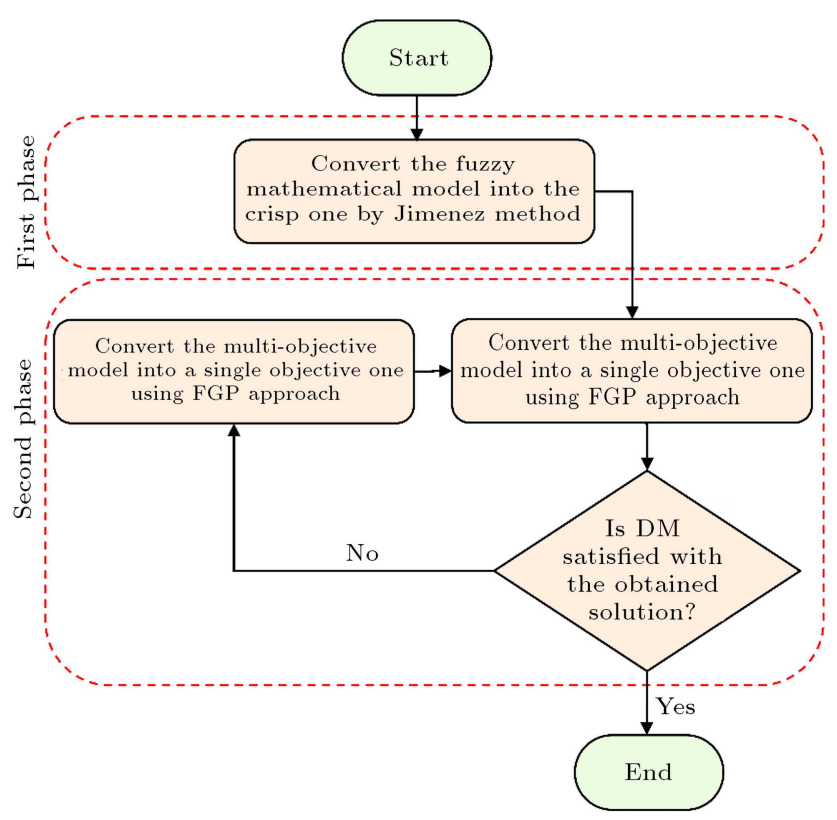

Figure 2. Flowchart of the proposed solution method. 
$\min \quad Z_{2}=\sum_{i=0}^{n} \sum_{j=1}^{n+1} \sum_{l=1}^{\widehat{N}_{i j}} \sum_{k=1}^{N V} X_{i j}^{l k}\left(\frac{R_{i j}^{l p}+2 R_{i j}^{l m}+R_{i j}^{l o}}{4}\right)$.

Similarly, according to the equation, Constraints (7) to (10) and (15) are equal to the following auxiliary crisp constraints:

$$
\begin{aligned}
& T_{i}^{k}+s_{i}+X_{i j}^{l k}\left(\alpha\left(\frac{t_{i j}^{l p}+t_{i j}^{l m}}{2}\right)+(1-\alpha)\left(\frac{t_{i j}^{l o}+t_{i j}^{l m}}{2}\right)\right) \\
&+t_{j}^{w}-T_{j}^{k} \leq\left(1-X_{i j}^{l k}\right) M \\
& i=1, \cdots, n ; \quad j=1, \cdots, n ; \quad k=1, \cdots, N V ; \\
& l=1, \cdots, \widehat{N}_{i j}, \\
& T_{i}^{k}+s_{i}+X_{i j}^{l k}\left(\alpha\left(\frac{t_{i j}^{l p}+t_{i j}^{l m}}{2}\right)+(1-\alpha)\left(\frac{t_{i j}^{l o}+t_{i j}^{l m}}{2}\right)\right) \\
&+t_{j}^{w}-T_{j}^{k} \leq\left(X_{i j}^{l k}-1\right) M \\
& i=1, \cdots, n ; \quad j=1, \cdots, n ; \quad k=1, \cdots, N V ; \\
& l=1, \cdots, \widehat{N}_{i j}, \\
& T_{D}^{k}+X_{o j}^{l k}\left(\alpha\left(\frac{t_{i j}^{l p}+t_{i j}^{l m}}{2}\right)+(1-\alpha)\left(\frac{t_{i j}^{l o}+t_{i j}^{l m}}{2}\right)\right) \\
&+t_{j}^{w}-T_{j}^{k} \leq\left(1-X_{o j}^{l k}\right) M \\
& i=1, \cdots, n ; \quad j=1, \cdots, n ; \quad k=1, \cdots, N V ; \\
&+t_{j}^{w}-T_{j}^{k} \geq\left(X_{o j}^{l k}-1\right) M \\
& i=1, \cdots, n ; \quad j=1, \cdots, n ; \quad k=1, \cdots, N V ; \\
& X_{i j}^{l k}\left(\alpha\left(\frac{R_{i j}^{l p}+R_{i j}^{l m}}{2}\right)+(1-\alpha)\left(\frac{R_{i j}^{l o}+R_{i j}^{l m}}{2}\right)\right) h_{i j} . \\
& T_{D}^{k}+X_{o j}^{l k}\left(\alpha\left(\frac{t_{i j}^{l p}+t_{i j}^{l m}}{2}\right)+(1-\alpha)\left(\frac{t_{i j}^{l o}+t_{i j}^{l m}}{2}\right)\right)
\end{aligned}
$$

As a results of using the interactive method proposed by Jimenez et al. [49], we are now confronted with multi objective mixed-integer linear programming. Certainly the above algorithm is used to solve the single-objective problem, because we are facing a two-objective mathematical model, so it is better to use another algorithm to solve the problem.

\subsection{FGP approach}

There are many exact methods such as weighted metric, weighted sum, $\varepsilon$-constraint, interactive approaches and, FGP for finding the optimal results of the multi-objective problems. In the real multiobjective problem, determining the specific values of priority objectives is so tricky. Because in most cases, the importance of the weight is uncertain or imprecise to the decision-maker point of view. On the other hand, the decision-making environment and the real relationship are the actual factors that determine the importance of the weight of the objective function. Therefore, the FGP approach is considered as one of the best tools to deal with these kinds of problems, and utilizing fuzzy theory sets in goal programming makes it possible for decision-makers to determine the vague aspiration levels $[51,52]$. In this study, because some inaccurate objectives conflict with each other, we applied the FGP method to convert multiple goals into a single objective [53]. Therefore, the objective functions and their corresponding constraints can be expressed as below:

$$
\begin{array}{ll}
\min & \sum_{i=1}^{k} w_{i} B_{i}^{+}, \\
& (\mathrm{AX})_{i}-t_{i}^{+} B_{i}^{+} \leq b_{i}, \\
\min & \sum_{i=1}^{k} w_{i} B_{i}^{-}, \\
& (\mathrm{AX})_{i}-t_{i}^{-} B_{i}^{-} \leq b_{i}, \\
\min & \sum_{i=1}^{k} w_{i}\left(B_{i}^{+}+B_{i}^{-}\right),
\end{array}
$$

where $\left(B_{i}^{+} / B_{i}^{-}\right)$is the permissible tolerance variable whose ratio is tolerance $\left(t_{i}^{+} / t_{i}^{-}\right)$. Also, $w_{i}$ shows the weight or importance of objective function $i$. Then, our final model is as follows [54]:

$$
\begin{aligned}
\min & \sum_{i=1}^{k} w_{i}\left(B_{i}^{+}+B_{i}^{-}\right), \\
& (\mathrm{AX})_{i}+t_{i}^{-} B_{i}^{+}=b_{i}, \quad i=1,2, \cdots, k, \\
& t_{i}^{-}, B_{i}^{-}, t_{i}^{+}, B_{i}^{+} \geq 0, \quad x_{j} \geq 0, \quad i=1,2, \cdots, n, \\
& \sum_{i=1}^{k} w_{i}=1 .
\end{aligned}
$$

Therefore, our proposed model can be transformed as follows:

$$
\min \sum_{i=1}^{k} w_{1} B_{1}^{+}+w_{2} B_{2}^{+}
$$


s.t.:

$$
\begin{aligned}
& \sum_{i=0}^{n} \sum_{j=1}^{n+1} \sum_{l=1}^{\widehat{N}_{i j}} \sum_{k=1}^{N V} X_{i j}^{l k} \widetilde{t}_{i j}^{1}+\sum_{i=1}^{n} t_{i}^{w}+t_{1}^{-} B_{1}^{-}-t_{1}^{+} B_{1}^{+}=b_{1} \\
& \sum_{i=0}^{n} \sum_{j=1}^{n+1} \sum_{l=1}^{\widehat{N}_{i j}} \sum_{k=1}^{N V} X_{i j}^{l k}\left(\widetilde{R A}_{i j}^{1}\left(k_{1} \widetilde{R E}_{i j}^{1}+k_{2} \widetilde{R P}_{i j}^{1}\right)\right) \\
& \quad+t_{2}^{-} B_{2}^{-}-t_{2}^{+} B_{2}^{+}=b_{2}
\end{aligned}
$$

other constraints:

$$
\begin{aligned}
& X_{i j}^{l k} \in\{0,1\} \quad t_{1}^{-}, t_{2}^{-}, B_{1}^{-}, B_{2}^{-}, t_{1}^{+}, t_{2}^{+}, B_{1}^{+}, B_{2}^{+} \geq 0 \\
& \sum_{i=1}^{k} w_{i}=1 .
\end{aligned}
$$

Also, the Zimmerman method [55] is applied to determine the membership functions associated with the different goals of the problem (minimizing problem):

$$
\mu_{i}=\left\{\begin{array}{ll}
1 & G_{i}(x) \leq g(i) \\
\frac{U_{i}-G_{i}(x)}{U_{i}-g(i)} & g(i) \leq G_{i}(x) \leq U_{i} \\
0 & G_{i}(x) \geq U_{i}
\end{array}\right\}
$$

where $g(i)$ presents the $i$ th goal and $G_{i}(x)$ shows the expressed level of the $i$ th goal. Also, $L_{i}$ and $U_{i}$ are lower tolerance limits and upper tolerance limits for $i$ th fuzzy goal, respectively. $\mu_{i}$ shows the membership function value of the goal within the interval $[0,1]$. If the goals are entirely achievable, the value of $\mu_{i}$ is 1 ; otherwise, when the goals are not achievable, it is zero.

\subsection{An IWO algorithm}

IWO algorithm proposed by Mehrabian and Lucas [56], simulates the colonization behavior of weeds. However, IWO is a numerical stochastic optimization algorithm that is inspired by the fact that natural invasive weeds are colonized based on weed biology and ecology. In this algorithm, a set of weeds is considered as an initial population. Every weed is a plant that grows in a specific area on a global scale, and humans cannot control or eliminate it. It is claimed that weeds always are the winner, and if more farmers try, more weeds will grow in the land. Weeds occupy the soil and produce new colonies based on the behavior of their pruning system. Through applying this system, weeds firstly find appropriate regions for attacking, invading, and generating new colonies. Therefore, new plants are generated for utilizing appropriate regions. Indeed, the colonizing behavior of the weeds is simulated by the IWO algorithm. This behavior can be applied as a method for solving optimization problems. Based on this method, the seeds will be scattered in a determined region and become weeds. These weeds start breeding. The weeds growing in the more fertile regions will have higher competency. Thus, higher breeding is reached in the vicinity of these weeds. At the same time, increasing the number of iterations will significantly reduce the distance between the generated weeds and their parent weeds. Moreover, among the existing weeds, the more capable weeds have a greater chance of survival. This process, called competitive elimination, will continue until better weeds are obtained. The steps of IWOA are described below. Besides, to a better understanding of the IWO approach, Figure 3 is depicted as the pseudocode.

- Generating an initial random population;

- Producing the seeds using the fitness value, as shown in Figure 4. The seeds can be produced according to the following formulation:

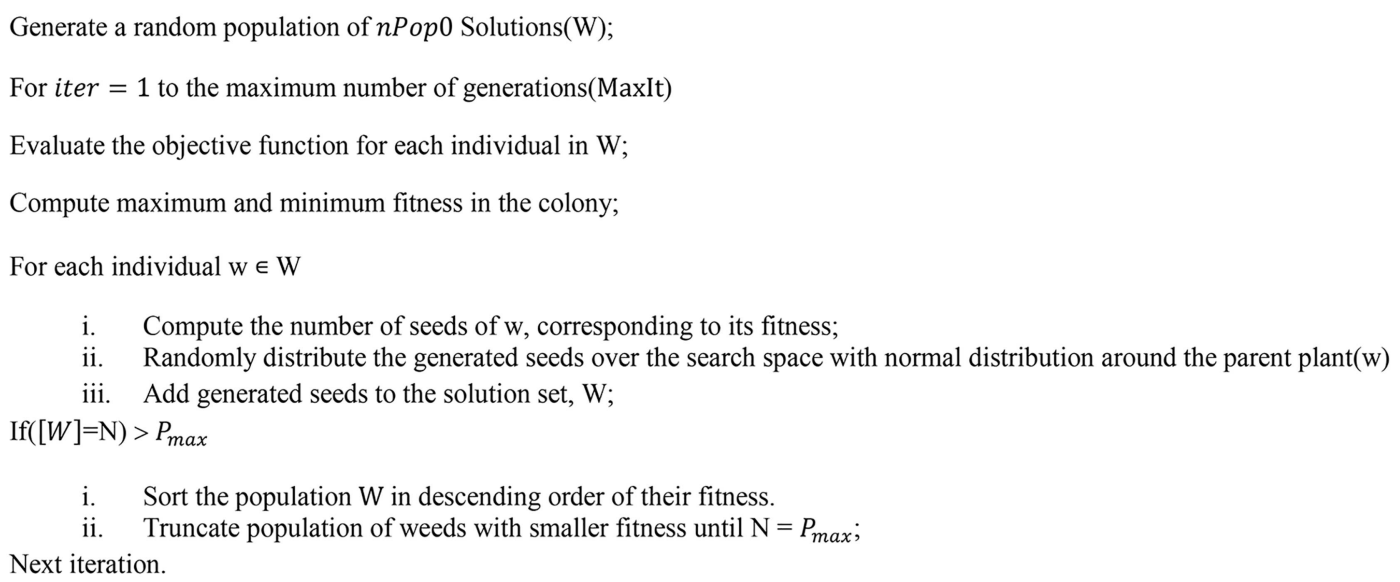

Figure 3. Pseudocode of Invasive Weeds Optimization (IWO) algorithm. 


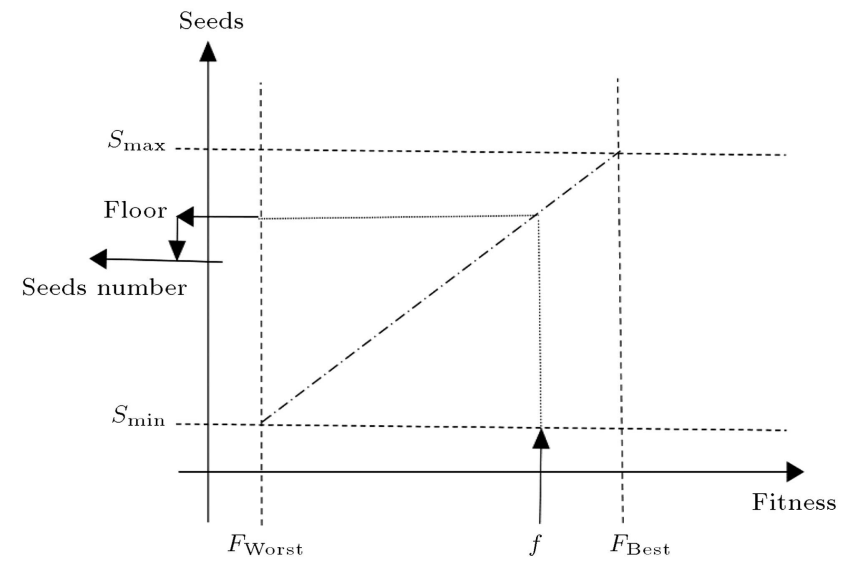

Figure 4. Producing seeds due to the fitness value.

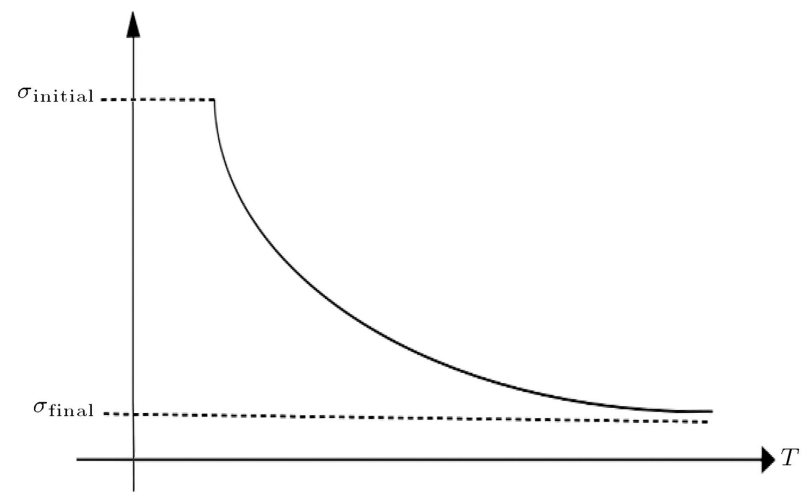

Figure 5. Standard deviation reduction.

$$
s=\left(S_{\min }+\left(S_{\max }-S_{\min }\right) \times\left(\frac{f-F_{\text {Worst }}}{F_{\text {Best }}-F_{\text {Worst }}}\right)\right)_{(26)}
$$

where, the best and worst fitness value, and the maximum and a minimum number of seeds generated by each weed are shown respectively by $F_{\text {Worst }}$, $F_{\text {Best }}, S_{\text {max }}$, and $S_{\text {min }}$;

- Children's seeds are distributed around the parent's seeds by Normal distribution, $\Delta x_{i} \sim N\left(0, \sigma_{t}^{2}\right)$. The standard deviation of the distribution gradually decreased over a period of time, as shown in Figure 3, and breading is performed using competency and updated standard deviation. The corresponding value of the standard deviation is determined by Eq. (27):

$$
\sigma_{t}=\left(\frac{T-t}{T}\right) \times\left(\sigma_{\text {initial }}-\sigma_{\text {final }}\right)+\sigma_{\text {final }},
$$

where, time, initial and final standard deviation of the seeds in each iteration are denoted respectively by $T, \sigma_{\text {final }}$, and $\sigma_{\text {final }}$ (see Figure 5 );

- Competitive elimination: If the total number of seeds reaches $P_{\max }$, sort the seeds and then eliminate the extra seeds (seeds with less fitness values);

- Control the stopping criterion.

\subsection{Genetic Algorithm (GA)}

One of the GA is a heuristic search that is inspired by Charles Darwin's theory of natural evolution. This algorithm reflects the process of natural selection that is, selecting the most suitable individuals to ensure the survival of the next generations. The process of natural selection starts with the selection of fittest individuals from a population. Their offspring and nextgeneration inherit the characteristics of their parents. If parents have better fitness, their children will be better than their parents and their chances of survival will be greater. This process continues to iterate, and eventually, the most suitable generation will be found. This notion can be applied to a search problem. We consider a set of solutions to a problem and choose the best set of solutions from them. Five phases are deemed to be discussed in a GA as follows:

1. Initial population: The procedure starts with many people, known as a Population. Everyone is an answer to a question that needs to be clarified. An individual is characterized by a set of parameters (variables) known as Genes. Genes are joined into a string to form a Chromosome (solution). In a $\mathrm{GA}$, the set of genes of an individual is represented using a string, according to the alphabet. Usually, binary values are used (a string of $1 \mathrm{~s}$ and $0 \mathrm{~s}$ ). We say that we encode the genes in a chromosome;

2. Fitness function: The fitness function determines how an individual adapts (the ability of an individual to compete with other individuals). It gives a fitness score to each individual. The probability that an individual will be selected for reproduction is based on its fitness score. The idea of the selection phase is to choose the fittest individuals and let them pass on their genes to the next generation. Two pairs of individuals (parents) are chosen based on their fitness scores. Individuals with high fitness have more chances to be selected for reproduction;

3. Crossover: Crossover is the most significant phase in a GA. For each pair of parents to be mated, a crossover point is chosen randomly from the the genes. Offspring are created by exchanging the genes of parents between themselves until the crossover point is reached. The new offspring are added to the population;

4. Mutation: When new offspring are formed, a low random chance exists that part of their genes would be mutated. This implies that some of the bits in the bit string can be flipped. A mutation occurs to maintain diversity within the population and prevent premature convergence. If the population converges (does not produce offspring that are significantly different from the previous generation), 
Generate an initial random population;

While iteration $<=$ maxiteration

iteration $=$ iteration +1

calculate the fitness of each individual

select the individuals according to their fitness

perform crossover with a probability $P_{c}$

perform mutation with probability $P_{m}$

end while

population $=$ selected individuals after crossover and mutation

Figure 6. Pseudocode of Genetic Algorithm (GA).

the algorithm terminates. Then it is said that the GA has provided a set of solutions to our problem. The population has a fixed size. With the formation of a new generation, the most unsuitable individuals die, providing space for new offspring. The sequence of phases repeats to produce individuals in each new generation, which are better than the previous generation. The pseudo-code of the GA algorithm is illustrated in Figure 6.

\section{Computational results}

In this section, first, the sensitivity of objective functions to the important input parameters is determined through several sensitivity analyses. Furthermore, two meta-heuristic algorithms (i.e., IWO and GA) are coded in MATLAB 2016a software to prove their performance efficiency in terms of the solution quality and calculating time. Finally, a real HAZMAT transportation case study in Iran is considered to confirm the validity and reliability of the proposed model and methods. Meanwhile, all computations were performed on a laptop with a $2.6-\mathrm{GHz} \mathrm{CPU}$ and $4 \mathrm{~GB}$ of RAM.

\subsection{Sensitive analysis}

In this section, using the information shown in Table 2, several small-scale test problems are analyzed to study the correctness of the proposed model and the efficiency of the proposed solution. First, the results of the objective function values obtained by the FGP method are summarized in Table 3 . Then, some sensitivity

Table 2. Data sets generated randomly.

\begin{tabular}{cccccc}
\hline \multirow{2}{*}{ Parameters } & \multicolumn{5}{c}{ Values } \\
\cline { 2 - 6 } & Problem 1 & Problem 2 & Problem 3 & Problem 4 & Problem 5 \\
\hline$i$ & 4 & 6 & 8 & 10 & 12 \\
$j$ & 1 & 1 & 1 & 1 & 1 \\
$l$ & 2 & 2 & 2 & 3 & 3 \\
$N V$ & 2 & 2 & 3 & 3 & 3 \\
$M V$ & 3 & 3 & 3 & 3 & 4 \\
$M R$ & 10 & 10 & 12 & 12 & 0.6 \\
$\alpha$ & 0.5 & 0.5 & 0.6 & 0.6 & 30 \\
$d_{i}$ & 10 & 15 & 20 & 25 & 60 \\
$C_{k}$ & 60 & 60 & 60 & 2.8 & 3.6 \\
$s_{i}$ & 0.5 & 1.6 & 2.3 & $\sim U(130,160)$ & $\sim U(180,220)$ \\
$a_{i}$ & $\sim U(20,30)$ & $\sim U(40,70)$ & $\sim U(80,110)$ & $\sim U(161,200)$ & $\sim U(221,260)$ \\
$b_{i}$ & $\sim U(31,50)$ & $\sim U(71,100)$ & $\sim U(111,150)$ & $\sim U(161)$ & $\sim U(105,130)$ \\
$\tilde{t}_{i j}^{l}$ & $\sim U(2,8)$ & $\sim U(15,25)$ & $\sim U(35,50)$ & $\sim U(73,90)$ & $\sim U(50,60)$ \\
$R_{i j}^{l k}$ & $\sim U(10,20)$ & $\sim U(20,30)$ & $\sim U(30,40)$ & $\sim U(40,50)$ &
\end{tabular}

Table 3. The summary results of tests examples solved by Fuzzy Goal Programming (FGP).

\begin{tabular}{|c|c|c|c|c|c|c|}
\hline \multirow{3}{*}{$\begin{array}{l}\text { Problem } \\
\text { no. }\end{array}$} & \multicolumn{4}{|c|}{ Proposed VRPTW model } & \multirow{2}{*}{\multicolumn{2}{|c|}{$\begin{array}{c}\text { Traditional } \\
\text { VRPTW mode }\end{array}$}} \\
\hline & \multicolumn{2}{|c|}{$\begin{array}{l}\text { Objective value } \\
\qquad(\alpha=0.95)\end{array}$} & \multicolumn{2}{|c|}{$\begin{array}{c}\text { Fuzzy goal } \\
\text { (upper tolerance limit }\left(U_{i}\right) \text { ) }\end{array}$} & & \\
\hline & $Z_{1}$ & $Z_{2}$ & $Z_{1}$ & $Z_{2}$ & $Z_{1}$ & $Z_{2}$ \\
\hline 1 & 72.8 & 8.2 & 76 & 11 & 88.9 & 12.8 \\
\hline 2 & 119.1 & 9.7 & 130 & 13 & 146.5 & 14.3 \\
\hline 3 & 120.0 & 13.5 & 133 & 19 & 149.0 & 21.8 \\
\hline 4 & 153.7 & 14.8 & 174 & 22 & 180.3 & 24.8 \\
\hline 5 & 141.4 & 16.8 & 163 & 27 & 172.8 & 29.6 \\
\hline
\end{tabular}


Table 4. The goal function degree value $\left(\mu_{i}\right)$ of tests examples.

\begin{tabular}{ccc}
\hline \multirow{2}{*}{$\begin{array}{c}\text { Problem } \\
\text { number }\end{array}$} & \multicolumn{2}{c}{ Goal function degree value $\left(\boldsymbol{\mu}_{\boldsymbol{i}}\right)$} \\
\cline { 2 - 3 } & $\begin{array}{c}\text { First goal } \\
\text { (total distribution }\end{array}$ & $\begin{array}{c}\text { Second goal } \\
\text { (total risks) }\end{array}$ \\
\hline 1 & 0.53 & 0.81 \\
2 & 0.72 & 0.62 \\
3 & 0.72 & 0.73 \\
4 & 0.84 & 0.82 \\
5 & 0.67 & 0.69 \\
\hline
\end{tabular}

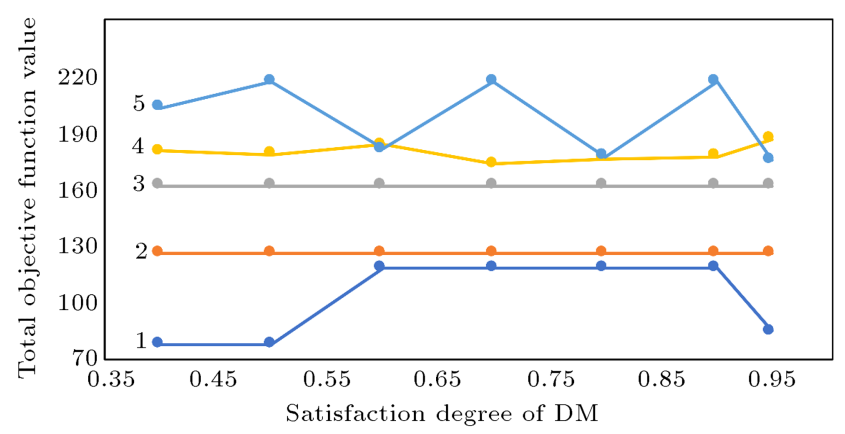

Figure 7. Total objective function value vs. mean of $\alpha$.

analyses are done on these small-size test problems using different values of $\propto$ to check the effect of these parameter values alterations on the objective functions. At the same time, due to the lack of acceptable examples and the proposed model features in the VRPTW literature, these examples were considered.

According to Table 3 , it is clear that when the problem is solved by FGP, the novelties proposed in the model produce better results than the previous traditional model. In other words, when we minimize the time and risk through the different contributions in this study, we can obtain better objective function values. Also, the function degree related to each goal is presented in Table 4 . The degree values of these functions are not equal to 1 , which means that due to the allowable tolerances, the relevant goals cannot be fully achieved, which seems reasonable. For example, the degree functions of the first and second goal for problem number 1 is equal to 0.53 and 0.81 which shows the average and high probability of achieving the goal. Other values of function degrees of Table 4 show that applying the FGP approach maximizes the access of each goal due to the decision-maker priorities.

Figure 7 shows the sensitivity analysis on the satisfaction degree $\alpha$ and its effect on the total objective function value $\left(Z_{T}\right)$ of problem number 1 . It is obvious that changing the $\alpha$-values has a significant effect on $Z_{T}$. The findings demonstrate that the optimal value of $Z_{T}$ (final solution based on FGP) can be obtained at different satisfaction degrees, depending on
Table 5. Check the objective functions of conflict.

\begin{tabular}{cccc}
\hline $\begin{array}{c}\text { Problem } \\
\text { number }\end{array}$ & $\begin{array}{c}\text { Objective } \\
\text { functions }\end{array}$ & $\boldsymbol{Z}_{\mathbf{1}}$ & $\boldsymbol{Z}_{\mathbf{2}}$ \\
\hline 1 & $Z_{1}$ & 52.5 & 6.1 \\
& $Z_{2}$ & 59.9 & 2.5 \\
& & & \\
2 & $Z_{1}$ & 53.2 & 3.4 \\
& $Z_{2}$ & 58.3 & 4.7 \\
& & & \\
3 & $Z_{1}$ & 54.8 & 3.4 \\
& $Z_{2}$ & 58.9 & 4.7 \\
& & & \\
4 & $Z_{1}$ & 58.4 & 6.2 \\
& $Z_{2}$ & 125.0 & 3.4 \\
& & & \\
5 & $Z_{1}$ & 62.6 & 7.3 \\
& $Z_{2}$ & 134.8 & 5.9 \\
\hline
\end{tabular}

the problem dimension and its parameters. Therefore, setting parameter $\alpha$ is a necessary condition to achieve a suitable solution. In fact, when the value of $\alpha$ is suspended by the Decision Maker (DM) according to the objective function preference to obtain their better value, a better objective function value is achieved.

In order to investigate the conflict of objective functions and prove the accuracy of the multi-objective assumption of the proposed model, the sensitivity analysis is done on the first and second objective function values. In this section, problem number 1 in Table 2 is considered as a small-sized test problem (i.e., $|i| \times|j| \times|k| \times|l|=4 \times 1 \times 2 \times 2)$ and the proposed bi-objective model is solved separately as two singleobjective problems. This means that the values of these two objective functions are determined once based on the first objective function (minimizing $Z_{1}$ ) and are calculated once using the second objective function (minimizing $Z_{2}$ ). Therefore, every objective function is coded in GAMS 22.9 software and solved by a CPLEX solver using the randomly generated data set shown in Table 2 .

The obtained solutions are shown in Table 5 . Obviously, a better value of one objective function will lead to the worst value of another objective function, which means that when one objective function improves, the other objective function becomes worse. Without considering the factors considered in other objective functions, the optimal value obtained for the first objective function is focused on reducing the distribution time including travel and waiting time. Therefore, it is logical to consider using the shortest route with high RPN and short travel time to transport dangerous products. This is why minimizing the total time increases the total risk value.

Table 5 also illustrates the optimal value of the 
second objective function regardless of other objective function considerations. In this case, the problem tends to consider routing with fewer RPNs and so, several routes are eliminated. Therefore, there are limited numbers of routes through which vehicles could deliver HAZMAT into customer nodes even if in case of an encounter with high distribution time. Therefore, the greater the number of second objective functions, the fewer the number of first objective functions. As one, when the goal is to minimize the first objective function, the first and second objectives of problem number 1 have objective function values of 52.5 and 6.1 , respectively. However, when the goal is to minimize the second objective function, these values equal 59.5 and 2.5. Therefore, this proves the objective functions of conflicts and the accuracy of the multi-objective assumption of the present study.

Finally, the considered numerical examples are investigated in three scenarios:

1. Without time window assumption;

2. With time window assumption for $50 \%$ of nodes;

3. With time window assumption for all nodes to analyze the effect of time window constraints on $Z_{1}, Z_{2}$ and $Z_{T}$.

The results shown in Table 6 show that the imposed time window limit increases the objective function

Table 6. Sensitive analysis on time window.

\begin{tabular}{|c|c|c|c|c|}
\hline \multirow{2}{*}{$\begin{array}{c}\text { Problem } \\
\text { number }\end{array}$} & \multirow{2}{*}{$\begin{array}{l}\text { Objective } \\
\text { functions }\end{array}$} & \multicolumn{3}{|c|}{ Scenario number } \\
\hline & & 1 & 2 & 3 \\
\hline \multirow{3}{*}{ 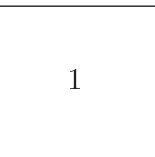 } & $Z_{1}$ & 18.7 & 56.2 & 61.8 \\
\hline & $Z_{2}$ & 7.0 & 7.3 & 7.1 \\
\hline & $Z_{T}$ & 33.6 & 69.4 & 78.0 \\
\hline \multirow{3}{*}{2} & $Z_{1}$ & 34.4 & 79.3 & 101.2 \\
\hline & $Z_{2}$ & 9.0 & 9.0 & 9.0 \\
\hline & $Z_{T}$ & 49.0 & 91.1 & 111.9 \\
\hline \multirow{3}{*}{3} & $Z_{1}$ & 78.8 & 73.6 & 102.0 \\
\hline & $Z_{2}$ & 12.5 & 12.5 & 12.5 \\
\hline & $Z_{T}$ & 39.2 & 104.4 & 131.1 \\
\hline \multirow{3}{*}{4} & $Z_{1}$ & 73.6 & 126.9 & 130.6 \\
\hline & $Z_{2}$ & 14.19 & 12.8 & 144.0 \\
\hline & $Z_{T}$ & 105.2 & 155.6 & 159.3 \\
\hline \multirow{3}{*}{5} & $Z_{1}$ & 82.4 & 117.9 & 126.6 \\
\hline & $Z_{2}$ & 98.1 & 17.2 & 132.2 \\
\hline & $Z_{T}$ & 119.9 & 129.1 & 163.1 \\
\hline
\end{tabular}

value of most nodes. When the problem-solving space is limited, it presents the normal operation of the proposed model.

\subsection{Managerial insight}

The accuracy of the presented mathematical model is verified through testing several numerical examples of the case study and the relevant results are provided in this section. In addition, the sensitivity of the model is investigated by changing the value of some important parameters.

\subsubsection{Performance evaluation}

In this section, through a comparative study using a large number of randomly generated data sets similar to Table 2, the performance of the proposed IWO is evaluated in terms of solution quality and calculation time. The mentioned generated data sets include 25 test problems ranging from small-sized to large-sized test problems and run for 5 replications (up to 100 nodes) to achieve a better analysis. In addition, since GA is one of the commonly used algorithms in the transportation of dangerous products, these algorithms are compared with the proposed algorithm to show the effectiveness of the proposed method. Meanwhile, the tuned parameters of IWO and GA are shown in Table 7.

To examine the notable differences between IWO and GA, Relative Percentage Deviation (RPD), Average Percentage of the Relative Gap (APRG), and the computational time (CPU) are considered as three comparisons metrics in the present study. RPD (\%) which is defined as the difference between the algorithm best quality solution and the best-known quality

Table 7. Invasive Weeds Optimization (IWO) and Genetic Algorithm (GA) parameter setting.

\begin{tabular}{clc}
\hline Algorithm & \multicolumn{1}{c}{ Parameter } & Value \\
\hline \multirow{4}{*}{ IWO } & MaxIt & 500 \\
& $P_{\max }$ & 1000 \\
& $\sigma_{\text {initial }}$ & 0.7 \\
& $\sigma_{\text {final }}$ & 0.6 \\
& $S_{\min }$ & 0.1 \\
& $S_{\max }$ & 10 \\
& & 100 \\
& Population size & 100 \\
& Crossover rate & 0.3 \\
GA & F & 0.8 \\
& Iteration in the & 10 \\
& initialization phase & \\
& Iteration in the & 200 \\
& initialization phase & \\
\hline
\end{tabular}


Table 8. Average Percentage of the Relative Gap (APRG), RPD, and CPU time for small-sized test problems in Invasive Weeds Optimization (IWO).

\begin{tabular}{|c|c|c|c|c|c|c|c|c|c|}
\hline \multirow{2}{*}{ Data set } & \multirow{2}{*}{ Exact time (s) } & \multicolumn{5}{|c|}{ Replications } & \multirow{2}{*}{ APRG (\%) } & \multirow{2}{*}{ RPD (\%) } & \multirow{2}{*}{ CPU (\%) } \\
\hline & & 1 & 2 & 3 & 4 & 5 & & & \\
\hline 1 & 30 & 0.00 & 0.00 & 0.00 & 0.00 & 0.00 & 0.000 & 0.00 & 19 \\
\hline 2 & 42 & 0.00 & 0.00 & 0.00 & 0.00 & 0.00 & 0.000 & 0.00 & 28 \\
\hline 3 & 65 & 0.02 & 0.01 & 0.02 & 0.01 & 0.01 & 0.014 & 0.02 & 36 \\
\hline 4 & 82 & 0.08 & 0.09 & 0.08 & 0.10 & 0.10 & 0.090 & 0.05 & 41 \\
\hline 5 & 123 & 0.48 & 0.45 & 0.43 & 0.52 & 0.52 & 0.480 & 0.10 & 45 \\
\hline 6 & 142 & 0.65 & 0.64 & 0.65 & 0.68 & 0.68 & 0.660 & 0.25 & 49 \\
\hline 7 & 181 & 1.00 & 1.12 & 1.12 & 1.05 & 1.01 & 1.060 & 0.10 & 51 \\
\hline 8 & 230 & 1.25 & 1.23 & 1.30 & 1.34 & 1.29 & 1.282 & 0.43 & 56 \\
\hline 9 & 346 & 1.40 & 1.32 & 1.33 & 1.35 & 1.38 & 1.356 & 0.65 & 59 \\
\hline 10 & 429 & 1.63 & 1.59 & 1.58 & 1.68 & 1.68 & 1.632 & 0.80 & 64 \\
\hline
\end{tabular}

Table 9. Average Percentage of the Relative Gap (APRG), RPD, and CPU time for small-sized test problems in Genetic Algorithm (GA).

\begin{tabular}{|c|c|c|c|c|c|c|c|c|c|}
\hline \multirow{2}{*}{ Data set } & \multirow{2}{*}{ Exact time (s) } & \multicolumn{5}{|c|}{ Replications } & \multirow{2}{*}{$\operatorname{AGP}(\%)$} & \multirow{2}{*}{ RPD (\%) } & \multirow{2}{*}{ CPU $(\%$} \\
\hline & & 1 & 2 & 3 & 4 & 5 & & & \\
\hline 1 & 30 & 0.00 & 0.00 & 0.00 & 0.00 & 0.00 & 0.000 & 0.00 & 20 \\
\hline 2 & 42 & 0.01 & 0.01 & 0.01 & 0.02 & 0.02 & 0.014 & 0.00 & 29 \\
\hline 3 & 65 & 0.04 & 0.05 & 0.04 & 0.06 & 0.05 & 0.048 & 0.05 & 38 \\
\hline 4 & 82 & 0.18 & 0.16 & 0.10 & 0.19 & 0.20 & 0.166 & 0.83 & 43 \\
\hline 5 & 123 & 0.68 & 0.65 & 0.70 & 0.65 & 0.67 & 0.670 & 1.04 & 47 \\
\hline 6 & 142 & 1.01 & 1.00 & 1.05 & 1.20 & 1.10 & 1.070 & 0.93 & 51 \\
\hline 7 & 181 & 1.25 & 1.18 & 1.20 & 1.15 & 1.20 & 1.196 & 2.22 & 54 \\
\hline 8 & 230 & 1.45 & 1.45 & 1.42 & 1.44 & 1.40 & 1.432 & 4.06 & 59 \\
\hline 9 & 346 & 1.73 & 1.70 & 1.68 & 1.72 & 1.70 & 1.706 & 3.12 & 61 \\
\hline 10 & 429 & 1.95 & 2.05 & 0.05 & 2.10 & 1.90 & 2.010 & 3.50 & 69 \\
\hline
\end{tabular}

solution of the problem in percent is estimated by:

$$
R P D=((\text { Best quality }- \text { Best known quality }) /
$$

Best known quality) $* 100 \%$,

and shown as "RPD" column in Tables 8 and 9. In addition, the computational results of IWO and GA illustrate the average relative gap percentage of the five replications of each small-sized problem (up to ten nodes) that are determined and reported as the "APRG" column in Tables 8 and 9. Therefore, the percentage of relative gap between IWO and GA with GAMS is calculated by $\left[100 \times\left(G_{O p t}-G_{A l g}\right) / G_{A l g}\right]$, where $G_{O p t}$ and $G_{A l g}$ are FGP objective function value obtained using GAMS software and solution obtained using the meta-heuristic algorithm, respectively. Also, the column "problem no." illustrates the number of problems in different dimensions which are generated based on Table 2.

The GAMS software cannot solve the proposed
NP-hard in the case of a larger test problem. Hence to solve the problem of large-sized samples, due to the Percentage of Relative Gap (PRG) measurement, only the comparison between IWO and GA is described in Table 10. In addition, the CPU time required by the proposed algorithm, and the fact that IWO is less than the CPU time required by the GA algorithm are shown in table $10 \mathrm{Also}$ this table shows that the method proposed in this study can improve CPU time. Therefore, the PRG between IWO and GA is calculated by $\left[100 \times\left(S_{G A}-S_{I W O}\right) / S_{G A}\right]$, where $S_{G A}$ and $S_{I W O}$ are the solution obtained from GA and IWO metaheuristics, respectively. The computational results of IWO and GA shown in Table 10 illustrate the average of the relative gap percentage and the required CPU for the five replications of each large-sized samples problem of the "APRG" and "exact time" column. As can be seen in Table 9, the minimum and maximum APRG between IWO and GA is $2.00 \%$ and $11.7 \%$, respectively.

Obviously, even on a small scale, the CPU time required to find the optimal solution increased expo- 
Table 10. Average Percentage of the Relative Gap (APRG), RPD, and CPU time of Invasive Weeds Optimization (IWO) in comparison to Genetic Algorithm (GA) for large-sized test problems.

\begin{tabular}{|c|c|c|c|c|c|c|c|c|c|}
\hline \multirow{3}{*}{ Data set } & \multirow{3}{*}{ Exact time } & \multirow{3}{*}{$\frac{\text { GA }}{\text { CPU (sec.) }}$} & \multirow{2}{*}{\multicolumn{5}{|c|}{$\begin{array}{c}\text { IWO } \\
\text { Replications } \\
\end{array}$}} & \multirow{3}{*}{ APRG (\%) } & \multirow{3}{*}{ CPU (sec) } \\
\hline & & & & & & & & & \\
\hline & & & 1 & 2 & 3 & 4 & 5 & & \\
\hline 11 & 671 & 72 & 2 & 2 & 1.9 & 2 & 1.98 & 2 & 65 \\
\hline 12 & 830 & 77 & 2.2 & 2.1 & 2.4 & 2.3 & 2.26 & 2.2 & 69 \\
\hline 13 & 1290 & 83 & 2.7 & 2.5 & 2.6 & 2.8 & 2.62 & 2.7 & 73 \\
\hline 14 & 1461 & 87 & 3.3 & 3.5 & 3.2 & 3.2 & 3.26 & 3.3 & 76 \\
\hline 15 & 1832 & 93 & 3.6 & 3.9 & 3.7 & 3.1 & 3.58 & 3.9 & 81 \\
\hline 16 & 2750 & 101 & 4.0 & 4.1 & 3.9 & 3.9 & 3.92 & 4 & 84 \\
\hline 17 & 4890 & 107 & 4.6 & 4.4 & 4.7 & 4.8 & 4.56 & 4.2 & 94 \\
\hline 18 & 6320 & 132 & 5.4 & 5.3 & 5.4 & 5.4 & 5.34 & 5.6 & 101 \\
\hline 19 & - & 151 & 5.9 & 5.7 & 5.8 & 6 & 5.84 & 5.9 & 110 \\
\hline 20 & - & 167 & 6.9 & 6.7 & 6.9 & 6.7 & 6.80 & 6.9 & 121 \\
\hline 21 & - & 204 & 7.7 & 7.8 & 7.6 & 7.9 & 7.70 & 7.1 & 132 \\
\hline 22 & - & 222 & 8.8 & 8.5 & 8.6 & 8.5 & 8.62 & 9.5 & 146 \\
\hline 23 & - & 272 & 9.5 & 9.4 & 9.2 & 9.9 & 9.46 & 10 & 153 \\
\hline 24 & - & 287 & 10.7 & 10.6 & 10.5 & 10.6 & 10.58 & 10.3 & 165 \\
\hline 25 & - & 301 & 11.7 & 11.5 & 11.7 & 11.5 & 11.54 & 11.7 & 172 \\
\hline
\end{tabular}

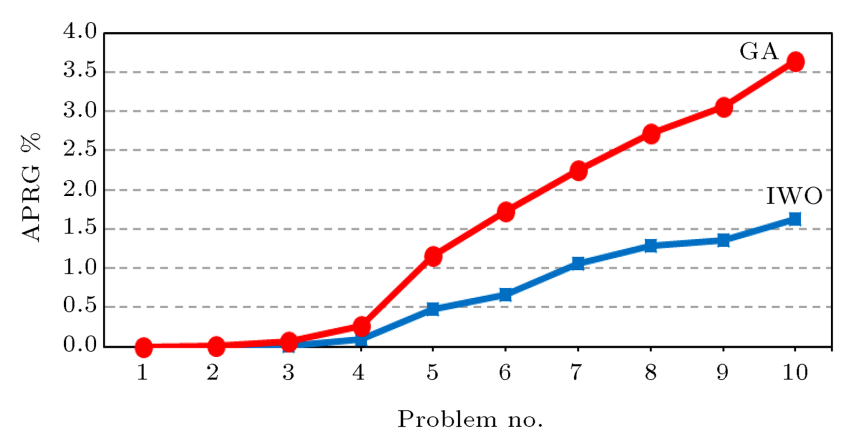

Figure 8. Average Percentage of the Relative Gap (APRG) of Invasive Weeds Optimization (IWO) and Genetic Algorithm (GA) in comparison to optimal solutions.

nentially. In addition, the column "IWO" illustrates the gaps of 5 replications, the average gap, and the average CPU time required for finding the near-optimal results. The results illustrated by Table 10 show that the Gams software is able for solving up to test instance 18 and this means finding optimal solutions for medium and large-sized instances is impossible by exact solutions. Also, the efficiency of the IWO method performance has been proved by the value, and that respectively shows the minimum, average and maximum percentage of a relative gap over all of the test problems for IWO. Besides, the last column illustrates the required CPU time for the IWO method and it illustrates that although the CPU time increased by the size of the problems, it is relatively small. Using the summary results provided in Figure 8 to Figure 10, both IWO and GA can find near-optimal solutions in a reasonable amount of time while IWO has a better

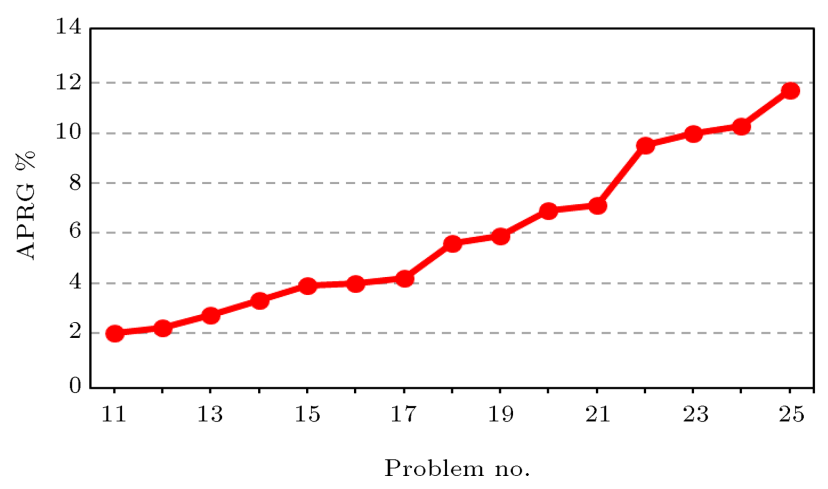

Figure 9. The computation time of Invasive Weeds Optimization (IWO) and Genetic Algorithm (GA).

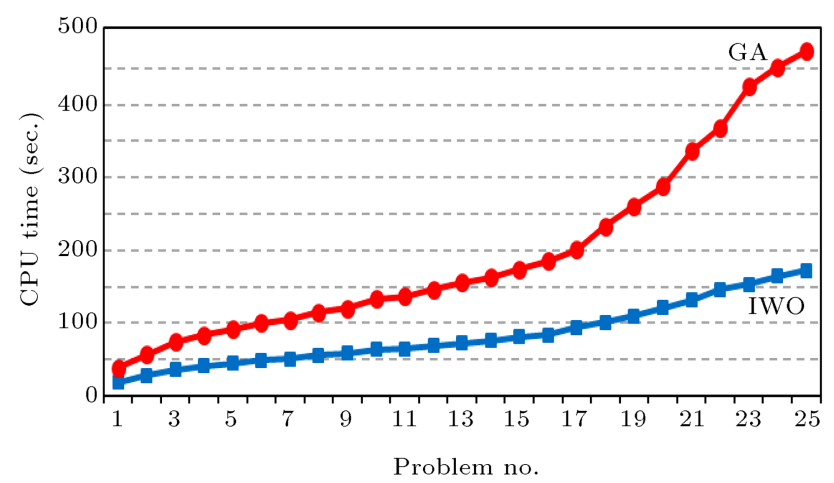

Figure 10. The computation time of Invasive Weeds Optimization (IWO) and Genetic Algorithm (GA).

performance in comparison to GA in terms of the solution quality. In addition to this, it is obvious that the exact CPU time has an exponential increase in comparison to the required CPU of IWO and GA. 


\subsubsection{Case study}

This section investigates a real case study related to hazardous fuel transportation of hazardous fuels in eastern Mazandaran province, northern Iran, to verify the performance of our mathematical model and the proposed method. The two main characteristics of northern Iran force government representatives and active companies in the distribution of dangerous products are considered to investigate the risk fairness. On the one hand, it is because this company is located in a tourist region. Many people travel to the region during weekends and statutory holidays in Iran. So, a small incident could lead to many financial, environmental, and environmental consequences. On the other hand, potential accidents and even unsafe distribution of HAZMAT in the region could affect the brand value of the manufacturing and consumer industries. In addition, the general sensitivity of the area and the potential risk of accidents when incidents related to the distribution of dangerous products occur will cause the police to impose some restrictions on the transportation of HAZMAT vehicles within certain hours or days. Therefore, parking the vehicle on the route may increase delivery delays and transportation costs. In the model proposed in this study, an attempt is made to introduce the best vehicle route and scheduling to reduce the total distribution time and the risks imposed on the transportation system.

In the present study, there is a distribution warehouse and 41 customer nodes the location of which is estimated by some experts and indicated in Figure 11. Meanwhile, the statistical data related to the problem are obtained from different sources to design the proposed model. Indeed, the proposed model needs two classified information:

1. Physical information including availability time of nodes, the service time of each node, capacity and number of vehicles, and available routes between two nodes. This information is collected from the qualified experts;

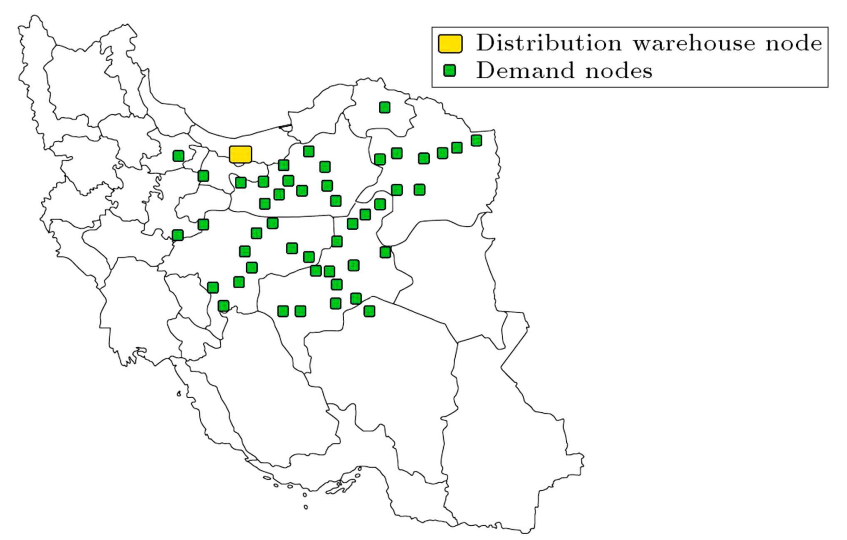

Figure 11. The location of the considered distribution warehouse node and demand nodes.
2. A team of 30 experts collected information about the fuzzy values of the question parameters, and they filled out 5 questionnaires using verbal variables.

Besides, the value of RPN can be calculated by the new framework proposed and described in Subsection 3.2. Also, 4 similar transportation vehicles were available for distribution, and the fuzzy values were considered. Figure 12 also shows the best transportation routes when the total times and risks are minimal.

In the present study, some sensitivity analysis is done on the case study under different values of $N V, d_{i}$, and $M V$ to realize how the objective function can be affected by these parameters values alterations. Figure 13 depicts the comparison of the changes of OFV1 and OFV2 with the changes of $N V$, respectively. Obviously, when the number of vehicles increases, the travel time of each vehicle decreases, and the total risk imposed on the system slightly increases. Because using more vehicles imposes more high-level risks on the transportation system, and as a consequence, RPN and OFV2 will be increased. Then, in this case, the value of OFV1 will decrease and the value of OFV2 will increase.

Figures 14 to 16, show variation of OFV1 and OFV2 compared to the variation of the $d_{i}$. Obviously,

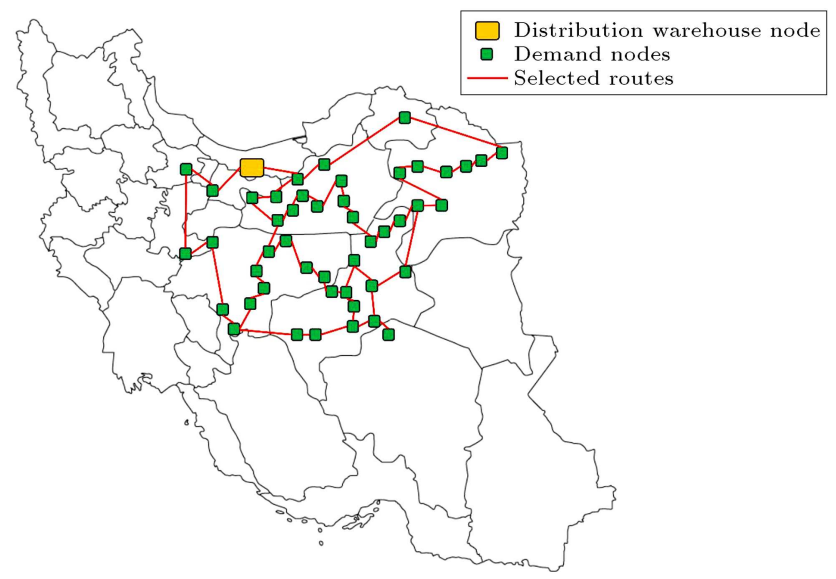

Figure 12. The best transportation routes due to minimizing risk and time.

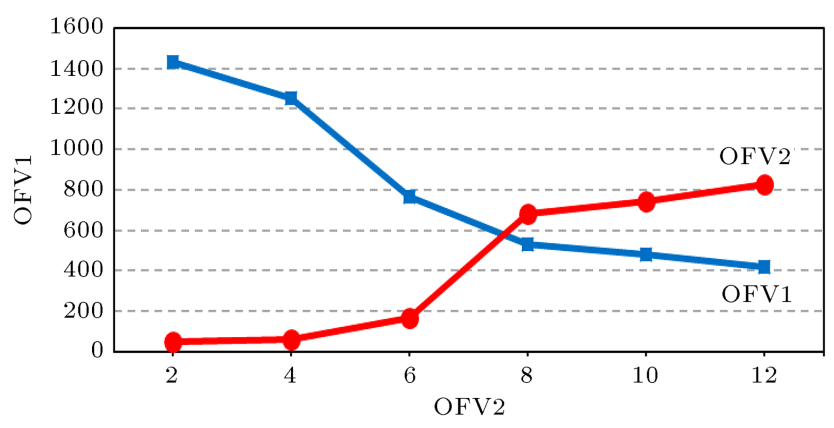

Figure 13. OFV1 and OFV2 vs. the number of vehicles. 


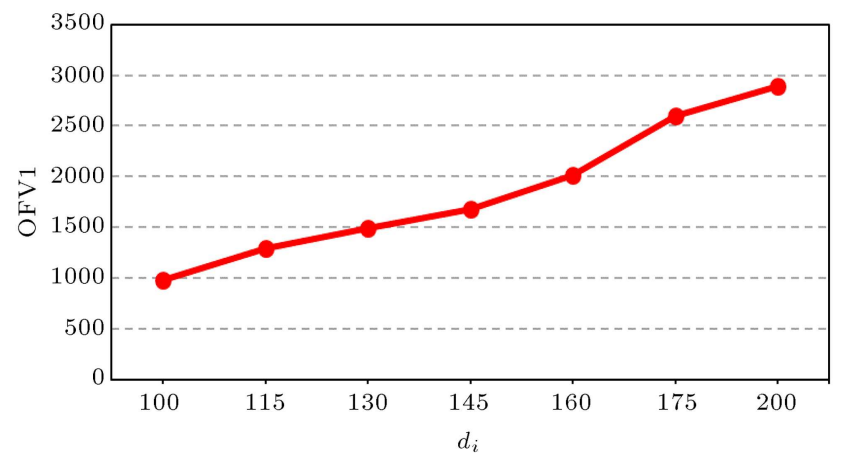

Figure 14. OFV1 vs. the amount of customer demand.

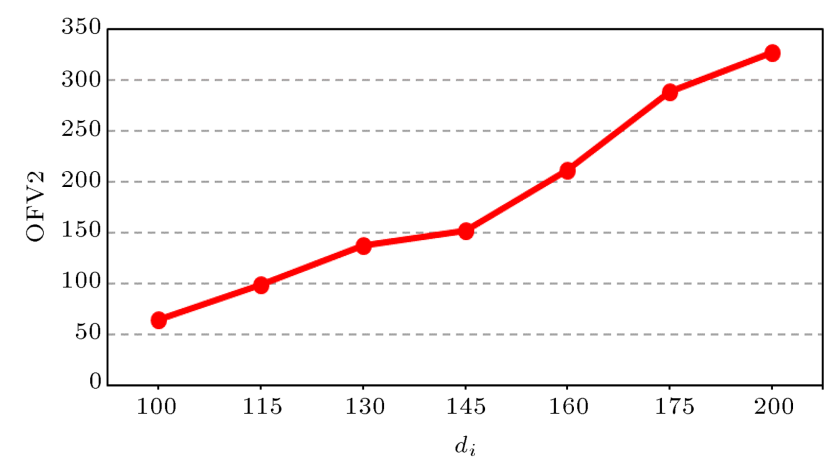

Figure 15. OFV1 vs. the amount of customer demand.

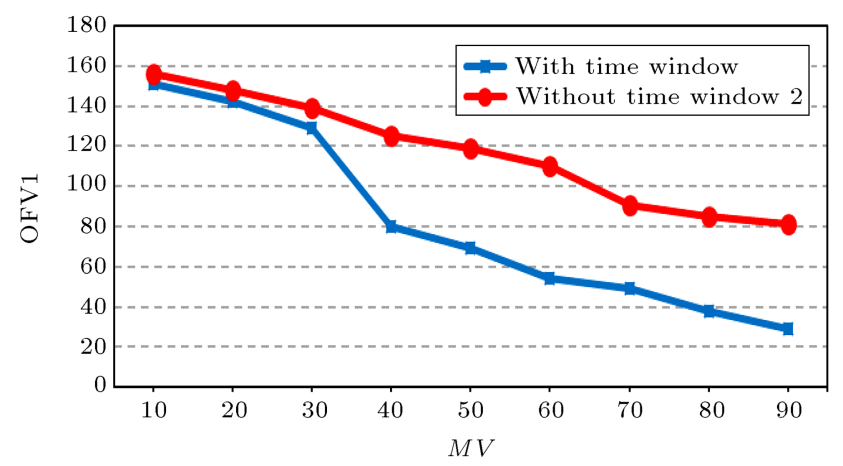

Figure 16. OFV1 vs. the maximum waiting time of vehicles at customers no.

the increase in customer demand leads to a worse value for achieving all objective functions. In other words, this is because increasing customer demand forces the model to use more vehicles for transportation. It will increase OFV1 due to the increase in travel time of using total vehicles. In addition, the use of more vehicles will bring more travel, and therefore will bring more high risks to the transportation system. Therefore, RPN and OFV2 will also be increased.

Figure 16 shows the change of OFV1 compared to the change of the maximum waiting time at the customer node to show the efficiency of the time window under consideration. It can be seen that two cases are considered (i.e., time window and no time window), and it is assumed that the $M V$ of each vehicle is the same. When considering the time window limit, the value of $M V$ is significantly smaller than the case that it is not considered in the model. Indeed, it is because the HAZMAT will be delivered to the customers in less waiting time and fewer congestion in case of considering time window limitation, total distribution time will be decreased substantially. In fact, less waiting time results in less distribution time, so OFV1 will be reduced.

\section{Conclusion and future research}

The main contributions of the presented paper are:

(i) Proposing a novel fuzzy bi-objective vehicle routing and scheduling model of HAZMAT transportation system for minimizing the total risk of HAZMAT transportation and maximizing the total Risk Priority Number (RPN) between two nodes;

(ii) Proposing a new framework of fuzzy Failure Mode and Effects Analysis (FMEA) and Fuzzy inference system to determine the high-level risks in calculating the total risks imposed to the of the hazardous material transportation system for the first time;

(iii) Using two meta-heuristics algorithms namely Invasive Weeds Optimization (IWO) and Genetic Algorithm (GA) to cope with the hardness of the large-sized problems.

Besides, to cope with the proposed bi-objective problem, at first, the Jimenez method was used to convert the fuzzy constraints of the proposed model into a crisp one. Then, a Fuzzy Goal Programming (FGP) method is used to convert it into a single objective one. In addition, some numerical large and medium-sized test problems were solved by GMAS software to verify the performance of the presented model and solution algorithm. The obtained solutions indicate that the objective function is sensitive to the satisfaction degree of the decision-maker and the time window limitation. Also, according to the obtained results, the proposed model can quickly reduce the total distribution time and cumulative risk of hazardous distribution. Also, a case study as a large-sized test problem in the northern part of Iran is solved by IWO and GA approaches, and through which the practicableness and effectiveness of the presented model are investigated. In summary, the results show that IWO requires less computing time to solve the problem and find a near-optimal solution, and has better performance than GA. In addition, it is found that the IWO algorithm converges faster than the GA algorithm.

There are some suggestions for future research. One suggestion can be to assume some fuzzy or possible parameters in the model, such as social and cultural 
factors, or to impose legal constraints that may affect the quality of the solution and the satisfaction of decision- makers. Another suggestion is to replace several HAZMAT types with only one type, and consider different vehicles with different capacities and various types of transportation in future studies. The final suggestion is to develop an accurate method like the Lagrangian relaxation algorithm, which seems to be a good direction for future research.

\section{References}

1. Ditta, A., Figueroa, O., Galindo, G., and Yie-Pinedo, R. "A review on research in transportation of hazardous materials", Socio-Economic Planning Sciences, 68, p. 100665 (2019).

2. US Census Bureau "United States: 2012, Hazardous Materials", United States, Department of Commerce, Retrieved from https://www.census.gov/content/ dam/Census/library/publications/2015/ econ/ec12tcf-us-hm.pdf (2015).

3. US Department of Transportation "INTELLIGENCE PORTALS. (n.d.)", Retrieved from https://portal. phmsa.dot.gov/analytics/saw.dll?

Portalpages\&amp;PortalPath $=\% 2$

Fshared\%2FPublic+Website+Pages $\% 2$ F_portal $\% 2$

F10+Year+Incident+Summary+ Reports, September 10 (2021).

4. Androutsopoulos, K.N. and Zografos, K.G. "Solving the bicriterion routing and scheduling problem for hazardous materials distribution", Transportation Research Part C: Emerging Technologies, 18(5), pp. 713726 (2010).

5. Pradhananga, R., Taniguchi, E., and Yamada, T. "Ant colony system based routing and scheduling for hazardous material transportation", Procedia-Social and Behavioral Sciences, 2(3), pp. 6097-6108 (2010).

6. Androutsopoulos, K.N. and Zografos, K.G. "A biobjective time-dependent vehicle routing and scheduling problem for hazardous materials distribution", EURO Journal on Transportation and Logistics, 1(12), pp. 157-183 (2012).

7. Govindan, K., Jafarian, A., Khodaverdi, R., and Devika, K. "Two-echelon multiple-vehicle locationrouting problem with time windows for optimization of sustainable supply chain network of perishable food", International Journal of Production Economics, 152, pp. 9-28 (2014).

8. Ghaderi, A. and Burdett, R.L. "An integrated location and routing approach for transporting hazardous materials in a bi-modal transportation network", Transportation Research Part E: Logistics and Transportation Review, 127, pp. 49-65 (2019).

9. Du, J., Li, X., Yu, L., Dan, R., and Zhou, J. "Multidepot vehicle routing problem for hazardous materials transportation: a fuzzy bilevel programming", Information Sciences, 399, pp. 201-218 (2017).
10. Ghaderi, A. and Burdett, R.L. "An integrated location and routing approach for transporting hazardous materials in a bi-modal transportation network", Transportation Research Part E: Logistics and Transportation Review, 127, pp. 49-65 (2019).

11. Pamučar, D., Ljubojević, S., Kostadinović, D., and Đorović, B. "Cost and risk aggregation in multiobjective route planning for hazardous materials transportation-A neuro-fuzzy and artificial bee colony approach", Expert Systems with Applications, 65, pp. 1-15 (2016).

12. Zero, L., Bersani, C., Paolucci, M., and Sacile, R. "Two new approaches for the bi-objective shortest path with a fuzzy objective applied to HAZMAT transportation", Journal of Hazardous Materials, 375, pp. 96-106 (2019).

13. Paydar, M.M., Babaveisi, V., and Safaei, A.S. "An engine oil closed-loop supply chain design considering collection risk", Computers \& Chemical Engineering, 104, pp. 38-55 (2017).

14. Talaei, M., Moghaddam, B.F., Pishvaee, M.S., Bozorgi-Amiri, A., and Gholamnejad, S. "A robust fuzzy optimization model for carbon-efficient closedloop supply chain network design problem: a numerical illustration in electronics industry", Journal of Cleaner Production, 113, pp. 662-673 (2016).

15. Kachitvichyanukul, V. "A particle swarm optimization for vehicle routing problem with time windows", Int. J. Oper. Res., 6(4), pp. 519-537 (2009).

16. Taniguchi, E., Thompson, E., Yamada, T., van Duin, J., and Logistics, C., Network Modelling and Intelligent Transport Systems, Emerald (2001).

17. Zhang, L., Guo, S., Zhu, Y., and Lim, A. "A tabu search algorithm for the safe transportation of hazardous materials", In Proceedings of the 2005 ACM Symposium on Applied Computing, pp. 940-946, ACM (2005).

18. Iassinovskaia, G., Limbourg, S., and Riane, F. "The inventory-routing problem of returnable transport items with time windows and simultaneous pickup and delivery in closed-loop supply chains", International Journal of Production Economics, 183, pp. 570-582 (2017).

19. Wang, N., Zhang, M., Che, A., and Jiang, B. "Biobjective vehicle routing for hazardous materials transportation with no vehicles travelling in echelon", IEEE Transactions on Intelligent Transportation Systems, 19(6), pp. 1867-1879 (2017).

20. Bula, G.A., Afsar, H.M., González, F.A., Prodhon, C., and Velasco, N. "Bi-objective vehicle routing problem for hazardous materials transportation", Journal of Cleaner Production, 206, pp. 976-986 (2019). 
21. Kassem, S. and Chen, M. "Solving reverse logistics vehicle routing problems with time windows", The International Journal of Advanced Manufacturing Technology, 68(1-4), pp. 57-68 (2013).

22. Zohal, M. and Soleimani, H. "Developing an ant colony approach for green closed-loop supply chain network design: a case study in gold industry", Journal of Cleaner Production, 133, pp. 314-337 (2016).

23. Paredes-Belmar, G., Bronfman, A., Marianov, V., and Latorre-Núñez, G. "Hazardous materials collection with multiple-product loading", Journal of Cleaner Production, 141, pp. 909-919 (2017).

24. Zografos, K.G. and Androutsopoulos, K.N. "A decision support system for integrated hazardous materials routing and emergency response decisions", Transportation Research Part C: Emerging Technologies, 16(6), pp. 684-703 (2008).

25. Talarico, L., Springael, J., Sörensen, K., and Talarico, F. "A large neighbourhood metaheuristic for the riskconstrained cash-in-transit vehicle routing problem", Computers \& Operations Research, 78, pp. 547-556 (2017).

26. Rabbani, M., Heidari, R., Farrokhi-Asl, H., and Rahimi, N. "Using metaheuristic algorithms to solve a multi-objective industrial hazardous waste location-routing problem considering incompatible waste types", Journal of Cleaner Production, 170, pp. 227-241 (2018).

27. Ma, C., Hao, W., Pan, F., and Xiang, W. "Road screening and distribution route multi-objective robust optimization for hazardous materials based on neural network and genetic algorithm", PLoS One, 13(6), e0198931 (2018).

28. Ma, C., Mao, B., Xu, Q., Hua, G., Zhang, S., and Zhang, T. "Multi-depot vehicle routing optimization considering energy consumption for hazardous materials transportation", Sustainability, 10(10), p. 3519 (2018).

29. López-Ramos, F., Nasini, S., and Guarnaschelli, A. "Road network pricing and design for ordinary and hazmat vehicles: Integrated model and specialized local search", Computers \& Operations Research, 109, pp. 170-187 (2019).

30. Jalili Ball, E., Tavakoli Moghaddam, R., and Javanshir, H. "Development of multi objective mathematical model of vehicle routing problem for transporting fuels by considering time windows and the environmental factors", Journal of Transportation Engineering, 8(3), pp. 343-354 (2015) (In Persian).

31. Alvarenga, G.B., Mateus, G.R., and De Tomi, G. "A genetic and set partitioning two-phase approach for the vehicle routing problem with time windows", Computers \& Operations Research, 34(6), pp. 15611584 (2007).
32. Erkut, E. and Verter, V. "Modeling of transport risk for hazardous materials", Operations Research, 46(5), pp. 625-642 (1998).

33. Marhavilas, P.K., Koulouriotis, D., and Gemeni, V. "Risk analysis and assessment methodologies in the work sites: On a review, classification and comparative study of the scientific literature of the period 20002009", Journal of Loss Prevention in the Process Industries, 24(5), pp. 477-523 (2011).

34. Bonvicini, S., Leonelli, P., and Spadoni, G. "Risk analysis of hazardous materials transportation: evaluating uncertainty by means of fuzzy logic", Journal of Hazardous Materials, 62(1), pp. 59-74 (1998).

35. Bubbico, R., Ferrari, C., and Mazzarotta, B. "Risk analysis of LPG transport by road and rail", Journal of Loss Prevention in the Process Industries, 13(1), pp. 27-31 (2000).

36. Topuz, E., Talinli, I., and Aydin, E. "Integration of environmental and human health risk assessment for industries using hazardous materials: a quantitative multi criteria approach for environmental decision makers", Environment International, 37(2), pp. 393403 (2011).

37. Jabbarzadeh, A., Azad, N., and Verma, M. "An optimization approach to planning rail hazmat shipments in the presence of random disruptions", Omega, 96, p. 102078 (2020).

38. Qureshi, A.G., Taniguchi, E., and Yamada, T. "A microsimulation based analysis of exact solution of dynamic vehicle routing with soft time windows", Procedia-Social and Behavioral Sciences, 39, pp. 205216 (2012).

39. Erkut, E. and Verter, V. "A framework for hazardous materials transport risk assessment", Risk Analysis, 15(5), pp. 589-601 (1995).

40. Abkowitz, M. and Cheng, P.D.M. "Developing a risk/cost framework for routing truck movements of hazardous materials", Accident Analysis \& Prevention, 20(1), pp. 39-51 (1988).

41. Hwang, S.T., Brown, D.F., O'Steen, J.K., Policastro, A.J., and Dunn, W.E. "Risk assessment for national transportation of selected hazardous materials", Transportation Research Record, 1763(1), pp. 114-124 (2001).

42. Rahimi, Y., Tavakkoli-Moghaddam, R., Iranmanesh, S.H., and Vaez-Alaei, M. "Hybrid approach to construction project risk management with simultaneous FMEA/ISO 31000/evolutionary algorithms: Empirical optimization study", Journal of Construction Engineering and Management, 144(6), p. 04018043 (2018).

43. Ho, C.C. and Chen, M.S. "Risk assessment and quality improvement of liquid waste management in Taiwan University chemical laboratories", Waste Management, 71, pp. 578-588 (2018). 
44. Hu, A.H., Hsu, C.W., Kuo, T.C., and Wu, W.C. "Risk evaluation of green components to hazardous substance using FMEA and FAHP", Expert Systems with Applications, 36(3), pp. 7142-7147 (2009).

45. Giannakis, M. and Papadopoulos, T. "Supply chain sustainability: A risk management approach", International Journal of Production Economics, 171, pp. 455-470 (2016).

46. Rebelo, A., Ferra, I., Gonçalves, I., and Marques, A.M. "A risk assessment model for water resources: releases of dangerous and hazardous substances", Journal of Environmental Management, 140, pp. 51-59 (2014).

47. Godoy, S.M., Santa Cruz, A.S., and Scenna, N.J. "STRRAP system-A software for hazardous materials risk assessment and safe distances calculation", Reliability Engineering \& System Safety, 92(7), pp. 847-857 (2007).

48. Wang, Y., Cai, Z., and Zhang, Q. "Enhancing the search ability of differential evolution through orthogonal crossover", Information Sciences, 185(1), pp. 153177 (2012).

49. Jiménez, M., Arenas, M., Bilbao, A., and Rodrı, M.V. "Linear programming with fuzzy parameters: an interactive method resolution", European Journal of Operational Research, 177(3), pp. 1599-1609 (2007).

50. Lenstra, J.K. and Kan, A.R. "Complexity of vehicle routing and scheduling problems", Networks, 11(2), pp. 221-227 (1981).

51. Yaghoobi, M.A. and Tamiz, M. "A method for solving fuzzy goal programming problems based on MINMAX approach", European Journal of Operational Research, 177(3), pp. 1580-1590 (2007).

52. Aköz, O. and Petrovic, D. "A fuzzy goal programming method with imprecise goal hierarchy", European Journal of Operational Research, 181(3), pp. 14271433 (2007).

53. Rabbani, M., Rahimi, Y., Kazemi, S.M., and Samavati, M. "Satisfying the energy demand of a rural area by considering the investment on renewable energy alternatives and depreciation costs", Journal of Renewable Energy, 2014, pp. 1-8 (2014).

54. Belmokaddem, M., Mekidiche, M., and Sahed, A. "Application of a fuzzy goal programming approach with different importance and priorities to aggregate production planning", Journal of Applied Quantitative Methods, 4(3), pp. 259-394 (2009).

55. Zimmermann, H.J. "Applications of fuzzy set theory to mathematical programming", Information Sciences, 36(1-2), pp. 29-58 (1985).

56. Mehrabian, A.R. and Lucas, C. "A novel numerical optimization algorithm inspired from weed colonization", Ecological Informatics, 1(4), pp. 355-366 (2006).

57. Aksen, D., Kaya, O., Salman, F.S., and Akça, Y. "Selective and periodic inventory routing problem for waste vegetable oil collection", Optimization Letters, 6(6), pp. 1063-1080 (2012).
58. Toumazis, I. and Kwon, C. "Routing hazardous materials on time-dependent networks using conditional value-at-risk", Transportation Research Part C: Emerging Technologies, 37, pp. 73-92 (2013).

59. Saat, M.R., Werth, C.J., Schaeffer, D., Yoon, H., and Barkan, C.P. "Environmental risk analysis of hazardous material rail transportation", Journal of Gazardous Materials, 264, pp. 560-569 (2014).

60. Ardjmand, E., Weckman, G., Park, N., Taherkhani, P., and Singh, M. "Applying genetic algorithm to a new location and routing model of hazardous materials", International Journal of Production Research, 53(3), pp. 916-928 (2015).

61. Timajchi, A., Al-e-Hashem, S.M.M., and Rekik, Y. "Inventory routing problem for hazardous and deteriorating items in the presence of accident risk with transshipment option", International Journal of Production Economics, 209, pp. 302-315 (2019).

62. Abuobidalla, O., Chen, M., and Chauhan, S. " A metaheuristic method for planning railway freight transportation with hazardous materials", Journal of Rail Transport Planning \& Management, 10, pp. 46-61 (2019).

\section{Biographies}

Aida Esmaeilidouki received her BSc in Industrial Engineering while working in various areas of this major, including mathematical modelling and optimization. She later completed her MSc in Industrial Engineering while publishing numerous peer-reviewed articles in the fields of mathematical modelling and multi-objective criteria decision making. She also obtained an MSAc in Mechanical Engineering from the University of British Columbia, and her thesis proposed a new framework consisted of simulation tools, machine learning, life cycle assessment, and multi-criteria decision making techniques. For the time being, she is a PhD student in Mechanical Engineering at University of British Columbia, where she is following her academic dream via integrating machine learning, mathematical modelling, and life cycle assessment methods to make manufacturing systems smart and sustainable.

Mehdi Mahzouni-Sani received his BSc degree in Electrical Engineering while he was working in many aspects of this major such as Power Systems Optimization, Reactive Power DispatchVolt/VAr Control Power Electrical Studies. He published some papers in the mentioned field of studies to see the practical effect of his knowledge on real case studies. Recently, he has been accepted as a prospective master student of Georgia Southern University and it sounds a great way for him to follow his dreams and academic activities. 
Abbas Nikhalat Jahromi is a Master graduate of Industrial Engineering. His Master thesis was about routing and scheduling of hazardous material. He has an investigation associated with hazardous material transportation which leads to outstanding and practical results in the real case study.
Fariborz Jolai is a Full Professor of the University of Tehran interested in theoretical and practical optimization research problems in service and manufacturing systems. His recent research works are on supply chain planning and management, health care system, and scheduling problems in uncertain environments. 